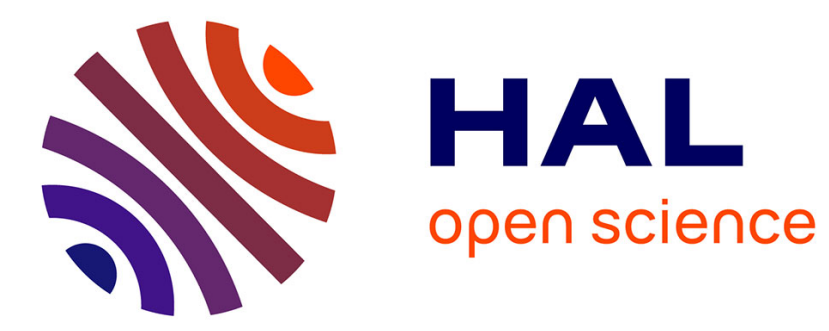

\title{
A Continuous Time Approach for the Asymptotic Value in Two-Person Zero-Sum Repeated Games
}

Pierre Cardaliaguet, Rida Laraki, Sylvain Sorin

\section{To cite this version:}

Pierre Cardaliaguet, Rida Laraki, Sylvain Sorin. A Continuous Time Approach for the Asymptotic Value in Two-Person Zero-Sum Repeated Games. SIAM Journal on Control and Optimization, 2012, 50, pp.1573-1596. 10.1137/110839473 . hal-00609476

\section{HAL Id: hal-00609476 https://hal.science/hal-00609476}

Submitted on 19 Jul 2011

HAL is a multi-disciplinary open access archive for the deposit and dissemination of scientific research documents, whether they are published or not. The documents may come from teaching and research institutions in France or abroad, or from public or private research centers.
L'archive ouverte pluridisciplinaire HAL, est destinée au dépôt et à la diffusion de documents scientifiques de niveau recherche, publiés ou non, émanant des établissements d'enseignement et de recherche français ou étrangers, des laboratoires publics ou privés. 


\title{
ECOLE POLYTECHNIQUE
}

CENTRE NATIONAL DE LA RECHERCHE SCIENTIFIQUE

\section{A Continuous Time Approach for the Asymptotic Value in Two-Person Zero-Sum Repeated Games}

\author{
Pierre Cardaliaguet \\ Rida Laraki \\ Sylvain Sorin
}

Juillet 2011

Cahier $n^{\circ}$ 2011- 11

\section{DEPARTEMENT D'ECONOMIE}

Route de Saclay

91128 PALAISEAU CEDEX

(33) 169333033

http://www.enseignement.polytechnique.fr/economie/

mailto:chantal.poujouly@polytechnique.edu 


\title{
A Continuous Time Approach for the Asymptotic Value in Two-Person Zero-Sum Repeated Games ${ }^{1}$
}

\author{
Pierre Cardaliaguet ${ }^{2}$ \\ Rida Laraki ${ }^{3}$ \\ Sylvain Sorin ${ }^{4}$
}

Juillet 2011

Cahier $n^{\circ}$ 2011- 11

\begin{abstract}
Résumé: Nous nous intéressons à la valeur asymptotique dans les jeux répétés à somme nulle avec une évaluation générale de la suite des paiements d'étapes. Nous montrons l'existence de la valeur asymptotique dans un sens robuste dans les jeux répétés à information incomplète, les jeux de splitting et les jeux absorbants. La technique de preuve consiste (1) à plonger le jeu répété en temps discret dans un jeu en temps continu et (2) à utiliser les solutions de viscosités.
\end{abstract}

\begin{abstract}
We consider the asymptotic value of two person zero-sum repeated games with general evaluations of the stream of stage payoffs. We show existence for incomplete information games, splitting games and absorbing games. The technique of proof consists in embedding the discrete repeated game into a continuous time one and to use viscosity solution tools.
\end{abstract}

Mots clés : Jeux stochastiques, jeux répétés, information incomplète, valeur asymptotique, principe de comparaison, inégalités variationnelles, solutions de viscosité, temps continu.

Key Words : $\quad$ Stochastic games, repeated games, incomplete information, asymptotic value, comparison principle, variational inequalities, viscosity solutions, continuous time.

Classification AMS: 91A15, 91A20, 93C41, 49J40, 58E35, 45B40, 35B51.

Classification JEL: C73, D82.

\footnotetext{
${ }^{1}$ This research was supported by grant ANR-10-BLAN 0112 (Cardaliaguet and Laraki) and ANR-08-BLAN0294-01 (Sorin).

${ }^{2}$ Ceremade, Université Paris-Dauphine, France. cardaliaguet@ceremade.dauphine.fr

${ }^{3}$ Economics Department of the Ecole Polytechnique and CNRS, France. rida.laraki@polytechnique.edu

${ }^{4}$ Combinatoire et Optimisation, IMJ, CNRS UMR 7586, Université Pierre et Marie Curie, and Laboratoire d'Economérie, Ecole Polytechnique, France. sorin@math.jussieu.fr
} 


\title{
A Continuous Time Approach for the Asymptotic Value in Two-Person Zero-Sum Repeated Games
}

\author{
Pierre Cardaliaguet*, Rida Laraki ${ }^{\dagger}$ and Sylvain Sorin ${ }^{\$}$
}

June 29, 2011

\begin{abstract}
We consider the asymptotic value of two person zero-sum repeated games with general evaluations of the stream of stage payoffs. We show existence for incomplete information games, splitting games and absorbing games. The technique of proof consists in embedding the discrete repeated game into a continuous time one and to use viscosity solution tools.
\end{abstract}

AMS classification: 91A15, 91A20, 93C41, 49J40, 58E35, 45B40, 35B51.

JEL classification: C73, D82.

Keywords: stochastic games, repeated games, incomplete information, asymptotic value, comparison principle, variational inequalities, viscosity solutions, continuous time.

\section{Introduction}

We study the asymptotic value of two person zero-sum repeated games. Our aim is to show that techniques which are typical of continuous time games ("viscosity solution") can be used to prove the convergence of the discounted value of such games as the discount factor tends to 0 , as well as the convergence of the value of the $n$-stage games as $n \rightarrow+\infty$ and to the same limit. The originality of our approach is that it provides the same proof for both classes of problems. It also allows to handle general decreasing evaluations of the stream of stage payoffs, as well as situations in which the payoff varies "slowly" in time. We illlustrate our purpose through three typical problems: repeated games with incomplete information on both sides, first analyzed by Mertens-Zamir (1971) [11], splitting games, considered by Laraki (2001) [6] and absorbing games, studied in particular by Kohlberg (1974) [5]. For the splitting games, we show in particular that the value of the $n$-stage game has a limit, which was not known yet.

In order to better explain our approach, we first recall the definition of Shapley operator for stochastic games, and its adaptation to games with incomplete information. Then we briefly describe the operator approach and its link with the viscosity solution techniques used in this paper.

\footnotetext{
*Ceremade, Université Paris-Dauphine, France. cardaliaguet@ceremade.dauphine.fr

${ }^{\dagger}$ CNRS, Economics Department, Ecole Polytechnique, France. rida.laraki@polytechnique.edu. Part time associated with Combinatoire et Optimisation, IMJ, CNRS UMR 7586, Université P. et M. Curie - Paris 6.

${ }^{\ddagger}$ Combinatoire et Optimisation, IMJ, CNRS UMR 7586, Faculté de Mathématiques, Université P. et M. Curie - Paris 6, Tour 15-16, 1 étage, 4 Place Jussieu, 75005 Paris and Laboratoire d'Econométrie, Ecole Polytechnique, France; sorin@math.jussieu.fr

$\S$ This research was supported by grant ANR-10-BLAN 0112 (Cardaliaguet and Laraki) and ANR-08-BLAN0294-01 (Sorin).
} 


\subsection{Discounted stochastic games and Shapley operator}

A stochastic game is a repeated game where the state changes from stage to stage according to a transition depending on the current state and the moves of the players. We consider the two person zero-sum case.

The game is specified by a state space $\Omega$, move sets $I$ and $J$, a transition probability $\rho$ from $I \times J \times \Omega \rightarrow \Delta(\Omega)$ and a payoff function $g$ from $I \times J \times \Omega \rightarrow \mathbb{R}$. All sets $A$ under consideration are finite and $\Delta(A)$ denotes the set of probabilities on $A$.

Inductively, at stage $n=1, \ldots$, knowing the past history $h_{n}=\left(\omega_{1}, i_{1}, j_{1}, \ldots ., i_{n-1}, j_{n-1}, \omega_{n}\right)$, player 1 chooses $i_{n} \in I$, player 2 chooses $j_{n} \in J$. The new state $\omega_{n+1} \in \Omega$ is drawn according to the probability distribution $\rho\left(i_{n}, j_{n}, \omega_{n}\right)$. The triplet $\left(i_{n}, j_{n}, \omega_{n+1}\right)$ is publicly announced and the situation is repeated. The payoff at stage $n$ is $g_{n}=g\left(i_{n}, j_{n}, \omega_{n}\right)$ and the total payoff is the discounted sum $\sum_{n} \lambda(1-\lambda)^{n-1} g_{n}$.

This discounted game has a value $v_{\lambda}$ (Shapley, 1953 [17]).

The Shapley operator $\mathbf{T}(\lambda, \cdot)$ associates to a function $f$ in $\mathbb{R}^{\Omega}$ the function $\mathbf{T}(\lambda, f)$ with :

$$
\mathbf{T}(\lambda, f)(\omega)=\operatorname{val}_{\Delta(I) \times \Delta(J)}\left[\lambda g(x, y, \omega)+(1-\lambda) \sum_{\tilde{\omega}} \rho(x, y, \omega)(\tilde{\omega}) f(\tilde{\omega})\right]
$$

where $g(x, y, \omega)=\mathrm{E}_{x, y} g(i, j, \omega)=\sum_{i, j} x_{i} y_{j} g(i, j, \omega)$ is the multilinear extension of $g(., ., \omega)$ and similarly for $\rho(., ., \omega)$, and val is the value operator

$$
\operatorname{val}_{\Delta(I) \times \Delta(J)}=\max _{x \in \Delta(I)} \min _{y \in \Delta(J)}=\min _{y \in \Delta(J)} \max _{x \in \Delta(I)} .
$$

The Shapley operator $\mathbf{T}(\lambda, \cdot)$ is well defined from $\mathbb{R}^{\Omega}$ to itself. Its unique fixed point is $v_{\lambda}$ (Shapley, 1953 [17]).

We will briefly write $(1)$ as $\mathbf{T}(\lambda, f)(\omega)=\operatorname{val}\{\lambda g+(1-\lambda) \mathrm{E} f\}$.

\subsection{Extension: repeated games}

A recursive structure leading to an equation similar to the previous one (1) holds in general for repeated games described as follows:

$M$ is a parameter space and $g$ a function from $I \times J \times M$ to $\mathbb{R}$. For each $m \in M$ this defines a two person zero-sum game with action spaces $I$ and $J$ for Player 1 and 2 respectively and payoff function $g(., ., m)$. The initial parameter $m_{1}$ is chosen at random and the players receive some initial information about it, say $a_{1}$ (resp. $b_{1}$ ) for player 1 (resp. player 2). This choice is performed according to some initial probability $\pi$ on $A \times B \times M$, where $A$ and $B$ are the signal sets of both players. At each stage $n$, player 1 (resp. 2) chooses an action $i_{n} \in I$ (resp. $\left.j_{n} \in J\right)$. This determines a stage payoff $g_{n}=g\left(i_{n}, j_{n}, m_{n}\right)$, where $m_{n}$ is the current value of the parameter. Then a new value of the parameter is selected and the players get some information. This is generated by a map $\rho$ from $I \times J \times M$ to probabilities on $A \times B \times M$. Hence at stage $n$ a triple $\left(a_{n+1}, b_{n+1}, m_{n+1}\right)$ is chosen according to the distribution $\rho\left(i_{n}, j_{n}, m_{n}\right)$. The new parameter is $m_{n+1}$, and the signal $a_{n+1}$ (resp. $b_{n+1}$ ) is transmitted to player 1 (resp. player 2). Note that each signal may reveal some information about the previous choice of actions $\left(i_{n}, j_{n}\right)$ and both the previous $\left(m_{n}\right)$ and the new $\left(m_{n+1}\right)$ values of the parameter.

Stochastic games correspond to public signals including the parameter.

Incomplete information games correspond to an absorbing transition on the parameter (which thus remains fixed) and no further information (after the initial one) on the parameter.

Mertens, Sorin and Zamir (1994) [12] Section IV.3, associate to each such repeated game $G$ an auxiliary stochastic game $\Gamma$ having the same values that satisfy a recursive equation of the type (1). However the play, hence the strategies in both games differ.

More precisely, in games with incomplete information on both sides, $M$ is a product space $K \times L$, 
$\pi$ is a product probability $p \otimes q$ with $p \in P=\Delta(K), q \in Q=\Delta(L)$ and in addition $a_{1}=k$ and $b_{1}=\ell$. Given the parameter $m=(k, \ell)$, each player knows his own component and holds a prior on the other player's component. From stage 1 on, the parameter is fixed and the information of the players after stage $n$ is $a_{n+1}=b_{n+1}=\left\{i_{n}, j_{n}\right\}$.

The auxiliary stochastic game $\Gamma$ corresponding to the recursive structure can be taken as follows: the "state space" $\Omega$ is $P \times Q$ and is interpreted as the space of beliefs on the true parameter.

$\mathbf{X}=\Delta(I)^{K}$ and $\mathbf{Y}=\Delta(J)^{L}$ are the type-dependent mixed action sets of the players; $g$ is extended on $\mathbf{X} \times \mathbf{Y} \times P \times Q$ by $g(x, y, p, q)=\sum_{k, \ell} p^{k} q^{\ell} g\left(x^{k}, y^{\ell}, k, \ell\right)$.

Given $(x, y, p, q) \in \mathbf{X} \times \mathbf{Y} \times P \times Q$, let $x(i)=\sum_{k} x_{i}^{k} p^{k}$ be the probability of action $i$ and $p(i)$ be the conditional probability on $K$ given the action $i$, explicitly $p^{k}(i)=\frac{p^{k} x_{i}^{k}}{x(i)}$ (and similarly for $y$ and $q$ ).

In this framework the Shapley operator is defined on the set $\mathcal{F}$ of continuous concave-convex functions on $P \times Q$ by:

$$
\mathbf{T}(\lambda, f)(p, q)=\operatorname{val}_{\mathbf{X} \times \mathbf{Y}}\left\{\lambda g(x, y, p, q)+(1-\lambda) \sum_{i, j} x(i) y(j) f(p(i), q(j))\right\}
$$

which is the new formulation of $\mathbf{T}(\lambda, f)(\omega)=\operatorname{val}\{\lambda g+(1-\lambda) \mathrm{E} f\}$ and the discounted value $v_{\lambda}(p, q)$ is the unique fixed point of $\mathbf{T}(\lambda,$.$) on \mathcal{F}$. These relations are due to Aumann and Maschler (1966) [1] and Mertens and Zamir (1971) [11].

\subsection{Extension: general evaluation}

The basic formula expressing the discounted value as a fixed point of the Shapley operator

$$
v_{\lambda}=\mathbf{T}\left(\lambda, v_{\lambda}\right)
$$

can be extended for values of games with the same plays but alternative evaluations of the stream of payoffs $\left\{g_{n}\right\}$.

For example the $n$-stage game, with payoff defined by the Cesaro mean $\frac{1}{n} \sum_{m=1}^{n} g_{m}$ of the stage payoffs, has a value $v_{n}$ and the recursive formula for the corresponding family of values is obtained similarly as

$$
v_{n}=\mathbf{T}\left(\frac{1}{n}, v_{n-1}\right)
$$

with obviously $v_{0}=0$.

Consider now an arbitrary evaluation probability $\mu$ on $\mathbb{I N}^{\star}$. The associated payoff in the game is $\sum_{n} \mu_{n} g_{n}$. Note that $\mu$ induces a partition $\Pi=\left\{t_{n}\right\}$ of $[0,1]$ with $t_{0}=0, t_{n}=\sum_{m=1}^{n} \mu_{m}, \ldots$ and thus the repeated game is naturally represented as a game played between times 0 and 1 , where the actions are constant on each subinterval $\left(t_{n-1}, t_{n}\right)$ which length $\mu_{n}$ is the weight of stage $n$ in the original game. Let $v_{\Pi}$ be its value. The corresponding recursive equation is now

$$
v_{\Pi}=\operatorname{val}\left\{t_{1} g_{1}+\left(1-t_{1}\right) \mathrm{E} v_{\Pi_{t_{1}}}\right\}
$$

where $\Pi_{t_{1}}$ is the normalization on $[0,1]$ of the trace of the partition $\Pi$ on the interval $\left[t_{1}, 1\right]$.

If one defines $V_{\Pi}\left(t_{n}\right)$ as the value of the game starting at time $t_{n}$, i.e. with evaluation $\mu_{n+m}$ for the payoff $g_{m}$ at stage $m$, one obtains the alternative recursive formula

$$
V_{\Pi}\left(t_{n}\right)=\operatorname{val}\left\{\mu_{n+1} g_{1}+\mathrm{E} V_{\Pi}\left(t_{n+1}\right)\right\} .
$$

The stationarity properties of the game form in terms of payoffs and dynamics induce time homogeneity

$$
V_{\Pi}\left(t_{n}\right)=\left(1-t_{n}\right) V_{\Pi_{t_{n}}}(0)
$$

where, as above, $\Pi_{t_{n}}$ stands for the normalization of $\Pi$ restricted to the interval $\left[t_{n}, 1\right]$. By taking the linear extension of $V_{\Pi}\left(t_{n}\right)$ we define for every partition $\Pi$, a function $V_{\Pi}(t)$ on $[0,1]$. 
Lemma 1 Assume that the sequence $\mu_{n}$ is decreasing. Then $V_{\Pi}$ is $C$-Lipschitz in $t$, where $C$ is a uniform bound on the payoffs in the game.

Proof. Given a pair of strategies $(\sigma, \tau)$ in the game $G$ with evaluation $\Pi$ starting at time $t_{n}$ in state $\omega$, the total payoff can be written in the form

$$
E_{\sigma, \tau}^{\omega}\left[\mu_{n+1} g_{1}+\ldots+\mu_{n+k} g_{k}+\ldots\right]
$$

where $g_{k}$ is the payoff at stage $k$. Assume now that $\sigma$ is optimal in the game $G$ with evaluation $\Pi$ starting at time $t_{n+1}$ in state $\omega$, then the alternative evaluation of the stream of payoffs satisfies, for all $\tau$

$$
E_{\sigma, \tau}^{\omega}\left[\mu_{n+2} g_{1}+\ldots+\mu_{n+k+1} g_{k}+\ldots\right] \geq V_{\Pi}\left(t_{n+1}, \omega\right)
$$

It follows that

$$
V_{\Pi}\left(t_{n}, \omega\right) \geq V_{\Pi}\left(t_{n+1}, \omega\right)-\left|E_{\sigma, \tau}^{\omega}\left[\left(\mu_{n+1}-\mu_{n+2}\right) g_{1}+\ldots+\left(\mu_{n+k}-\mu_{n+k+1}\right) g_{k}+\ldots\right]\right|
$$

hence $\mu_{n}$ being decreasing

$$
V_{\Pi}\left(t_{n}, \omega\right) \geq V_{\Pi}\left(t_{n+1}, \omega\right)-\mu_{n+1} C .
$$

This and the dual inequality imply that the linear interpolation $V_{\Pi}(., \omega)$ is a $C$ Lipschitz function in $t$.

\subsection{Asymptotic analysis: previous results}

We consider now the asymptotic behavior of $v_{n}$ as $n$ goes to $\infty$, or of $v_{\lambda}$ as $\lambda$ goes to 0 .

For games with incomplete information on one side, the first proofs of the existence of $\lim _{n \rightarrow \infty} v_{n}$ and $\lim _{\lambda \rightarrow 0} v_{\lambda}$ are due to Aumann and Maschler (1966) [1], including in addition an identification of the limit as $\operatorname{Cav}_{\Delta(K)} u$. Here $u(p)=\operatorname{val}_{\Delta(I) \times \Delta(J)} \sum_{k} p^{k} g(x, y, k)$ is the value of the one shot non revealing game, where the informed player does not use his information and $\operatorname{Cav}_{C}$ is the concavification operator: given $\phi$, a real bounded function defined on a convex set $C$, $\operatorname{Cav}_{C}(\phi)$ is the smallest function greater than $\phi$ and concave, on $C$.

Extensions of these results to games with lack of information on both sides were achieved by Mertens and Zamir (1971) [11]. In addition they identified the limit as the only solution of the system of implicit functional equations with unknown $\phi$ :

$$
\begin{gathered}
\phi(p, q)=\operatorname{Cav}_{p \in \Delta(K)} \min \{\phi, u\}(p, q), \\
\phi(p, q)=\operatorname{Vex}_{q \in \Delta(L)} \max \{\phi, u\}(p, q)
\end{gathered}
$$

Here again $u$ stands for the value of the non revealing game:

$$
u(p, q)=\operatorname{val}_{\Delta(I) \times \Delta(J)} \sum_{k, \ell} p^{k} q^{\ell} g(x, y, k, \ell)
$$

and $\mathbf{M Z}$ will denote the corresponding operator

$$
\phi=\mathbf{M Z}(u) .
$$

As for stochastic games, the existence of $\lim _{\lambda \rightarrow 0} v_{\lambda}$ is due to Bewley and Kohlberg (1976) [3] using algebraic arguments: the Shapley fixed point equation can be written as a finite set of polynomial inequalities involving the variables $\left\{\lambda, x_{\lambda}(\omega), y_{\lambda}(\omega), v_{\lambda}(\omega) ; \omega \in \Omega\right\}$ thus it defines a semi-algebraic set in some Euclidean space $\mathbb{R}^{N}$, hence by projection $v_{\lambda}$ has an expansion in Puiseux series of $\lambda$.

The existence of $\lim _{n \rightarrow \infty} v_{n}$ is obtained by an algebraic comparison argument, Bewley and Kohlberg (1976) [4].

The asymptotic values for specific classes of absorbing games with incomplete information are studied in Sorin (1984), [18], (1985) [19], see also Mertens, Sorin and Zamir (1994) [12]. 


\subsection{Asymptotic analysis: operator approach and comparison criteria}

Starting with Rosenberg and Sorin (2001) [15] several existence results for the asymptotic value have been obtained, based on the Shapley operator: continuous moves absorbing and recursive games, games with incomplete information on both sides, and for absorbing games with incomplete information on one side, Rosenberg (2000) [14].

We describe here an approach that was initially introduced by Laraki (2001) [6] for the discounted case. The analysis of the asymptotic behavior for the discounted games is simpler because of its stationarity: $v_{\lambda}$ is a fixed point of (3). Various discounted game models have been solved using a variational approach (see Laraki [6], [7] and [10]).

Our work is the natural extension of this analysis to more general evaluations of the stream of stage payoffs including the Cesaro mean and its limit. Recall that each such evaluation can be interpreted as a discretization of an underlying continuous time game. We prove for several classes of games (incomplete information, splitting, absorbing) the existence of a (uniform) limit of the values of the discretized continuous time game as the mesh of the discretization goes to zero. The basic recursive structure is used to formulate variational inequalities that have to be satisfied by any accumulation point of the sequences of values. Then an ad-hoc comparison principle allows to prove uniqueness, hence convergence. Note that this technique is a transposition to discrete games of the numerical schemes used to approximate the value function of differential games via viscosity solution arguments, as developed in Barles-Souganidis [2]. The difference is that: in differential games the dynamics is given in continuous time, hence the limit game is well defined and the question is the existence of its value while here we consider accumulation points of sequences of functions satisfying an adapted recursive equation which is not available in continuous time. Another main difference is that, in our case, the limit equation is singular and does not satisfy the conditions usually required to apply the comparison principles.

To sum up, the paper unifies tools used in discrete and continuous time approaches by dealing with functions defined on the product state $\times$ time space, in the spirit of Vieille (1992) [22] for weak approachability or Laraki (2002) [8] for the dual game of a repeated game with lack of information on one side, see also Sorin (2005) [21].

\section{Repeated Games with Incomplete Information}

Let us briefly recall the structure of repeated games with incomplete information: at the beginning of the game, the pair $(k, \ell)$ is chosen at random according to some product probability $p \otimes q$ where $p \in P=\Delta(K)$ and $q \in Q=\Delta(L)$. Player 1 knows $k$ while player 2 knows $\ell$. At each stage $n$ of the game, player 1 (resp. player 2) chooses a mixed strategy $x_{n} \in \mathbf{X}=(\Delta(I))^{K}$ (resp. $\left.y_{n} \in \mathbf{Y}=(\Delta(J))^{K}\right)$. This determines an expected payoff $g\left(x_{n}, y_{n}, p, q\right)$.

\subsection{The discounted game}

We now describe the analysis in the discounted case. The total payoff is given by the expectation of $\sum_{n} \lambda(1-\lambda)^{n} g\left(x_{n}, y_{n}, p, q\right)$ and the corresponding value $v_{\lambda}(p, q)$ is the unique fixed point of $\mathbf{T}(\lambda,).(2)$ on $\mathcal{F}([1],[11])$. In particular, $v_{\lambda}$ is concave in $p$ and convex in $q$.

We follow here Laraki (2001) [6].

Note that the family of functions $\left\{v_{\lambda}(p, q)\right\}$ is $C$-Lipschitz continuous, where $C$ is an uniform bound on the payoffs, hence relatively compact. To prove convergence it is enough to show that there is only one accumulation point (for the uniform convergence on $P \times Q$ ).

Remark that by (3) any accumulation point $w$ of the family $\left\{v_{\lambda}\right\}$ will satisfy

$$
w=\mathbf{T}(0, w)
$$


i.e. is a fixed point of the projective operator, see Sorin [20], appendix C.

Explicitly here: $\mathbf{T}(0, w)=\operatorname{val}_{\mathbf{X} \times \mathbf{Y}}\left\{\sum_{i, j} x(i) y(j) w(p(i), q(j))\right\}=\operatorname{val}_{\mathbf{X} \times \mathbf{Y}} \mathbf{E}_{x, y, p, q} w(\tilde{p}, \tilde{q})$, where $\tilde{p}=\left(p^{k}(i)\right)$ and $\tilde{q}=\left(q^{l}(j)\right)$.

Let $\mathcal{S}$ be the set of fixed points of $\mathbf{T}(0, \cdot)$ and $\mathcal{S}_{0} \subset \mathcal{S}$ the set of accumulation points of the family $\left\{v_{\lambda}\right\}$. Given $w \in \mathcal{S}_{0}$, we denote by $\mathbf{X}(p, q, w) \subseteq \mathbf{X}$ the set of optimal strategies for player 1 (resp. $\mathbf{Y}(p, q, w) \subseteq \mathbf{Y}$ for player 2) in the projective game with value $\mathbf{T}(0, w)$ at $(p, q)$. A strategy $x \in \mathbf{X}$ of player 1 is called non-revealing at $p, x \in N R_{\mathbf{X}}(p)$ if $\tilde{p}=p$ a.s. (i.e. $p(i)=p$ for all $i \in I$ with $x(i)>0)$ and similarly for $y \in \mathbf{Y}$. The value of the non revealing game satisfies

$$
u(p, q)=\operatorname{val}_{N R_{\mathbf{X}}(p) \times N R_{\mathbf{Y}}(q)} g(x, y, p, q) .
$$

A subset of strategies is non-revealing if all its elements are non-revealing.

Lemma 2 Let $w \in \mathcal{S}_{0}$ and $\mathbf{X}(p, q, w) \subset N R_{\mathbf{X}}(p)$ then

$$
w(p, q) \leq u(p, q) .
$$

Proof. Consider a family $\left\{v_{\lambda_{n}}\right\}$ converging to $w$ and $x_{n} \in \mathbf{X}$ optimal for $\mathbf{T}\left(\lambda_{n}, v_{\lambda_{n}}\right)(p, q)$, see (2). Jensen's inequality applied to (2) leads to

$$
v_{\lambda_{n}}(p, q) \leq \lambda_{n} g\left(x_{n}, j, p, q\right)+\left(1-\lambda_{n}\right) v_{\lambda_{n}}(p, q), \quad \forall j \in J .
$$

Thus

$$
v_{\lambda_{n}}(p, q) \leq g\left(x_{n}, j, p, q\right)
$$

If $\bar{x} \in \mathbf{X}$ is an accumulation point of the family $\left\{x_{n}\right\}$, then $\bar{x}$ is still optimal in $\mathbf{T}(0, w)(p, q)$. Since, by assumption $\mathbf{X}(p, q, w) \subset N R_{\mathbf{X}}(p), \bar{x}$ is non revealing and therefore one obtains as $\lambda_{n}$ goes to 0 :

$$
w(p, q) \leq g(\bar{x}, j, p, q), \quad \forall j \in J .
$$

So, by (9),

$$
w(p, q) \leq \max _{x \in N R_{\mathbf{X}}(p)} \min _{j \in J} g(x, j, p, q)=u(p, q) .
$$

Consider now $w_{1}$ and $w_{2}$ in $\mathcal{S}$ and let $\left(p_{0}, q_{0}\right)$ be an extreme point of the (convex hull of) the compact set in $P \times Q$ where the difference $\left(w_{1}-w_{2}\right)(p, q)$ is maximal (this argument goes back to Mertens-Zamir (1971) [11]).

\section{Lemma 3}

$$
\mathbf{X}\left(p_{0}, q_{0}, w_{1}\right) \subset N R_{\mathbf{X}}\left(p_{0}\right), \quad \mathbf{Y}\left(p_{0}, q_{0}, w_{2}\right) \subset N R_{\mathbf{Y}}\left(q_{0}\right) .
$$

Proof. By definition, if $x \in \mathbf{X}\left(p_{0}, q_{0}, w_{1}\right)$ and $y \in \mathbf{Y}\left(p_{0}, q_{0}, w_{2}\right)$,

$$
w_{1}\left(p_{0}, q_{0}\right) \leq \mathrm{E}_{x, y, p_{0}, q_{0}} w_{1}(\tilde{p}, \tilde{q})
$$

and

$$
w_{2}\left(p_{0}, q_{0}\right) \geq \mathrm{E}_{x, y, p_{0}, q_{0}} w_{2}(\tilde{p}, \tilde{q}) .
$$

Hence $(\tilde{p}, \tilde{q})$ belongs a.s. to the argmax of $w_{1}-w_{2}$ and the result follows from the extremality of $\left(p_{0}, q_{0}\right)$.

Proposition $4 \lim _{\lambda \rightarrow 0} v_{\lambda}$ exists. 
Proof. Let $w_{1}$ and $w_{2}$ be two different elements in $\mathcal{S}_{0}$ and suppose that $\max w_{1}-w_{2}>0$. Let $\left(p_{0}, q_{0}\right)$ be an extreme point of the (convex hull of) the compact set in $P \times Q$ where the difference $\left(w_{1}-w_{2}\right)(p, q)$ is maximal. Then Lemmas 2 and 3 imply $w_{1}\left(p_{0}, q_{0}\right) \leq u\left(p_{0}, q_{0}\right) \leq w_{2}\left(p_{0}, q_{0}\right)$, hence a contradiction. The convergence of the family $\left\{v_{\lambda}\right\}$ follows.

Given $w \in \mathcal{S}$ let $\mathcal{E} w(., q)$ be the set of $p \in P$ such that $(p, w(p, q))$ is an extreme point of the epigraph of $w(., q)$.

Lemma 5 Let $w \in \mathcal{S}$. Then $p \in \mathcal{E} w(., q)$ implies $\mathbf{X}(p, q, w) \subset N R_{\mathbf{X}}(p)$.

Proof. Use the fact that if $x \in \mathbf{X}(p, q, w)$ and $y \in N R_{\mathbf{Y}}(q)$

$$
w(p, q) \leq \mathrm{E}_{x, y, p, q} w(\tilde{p}, \tilde{q})=\mathrm{E}_{x, p} w(\tilde{p}, q) .
$$

Hence one recovers the characterization through the variational inequalities of Mertens and Zamir (1971) [11] and one identifies the limit as MZ (u).

Proposition $6 \lim _{\lambda \rightarrow 0} v_{\lambda}=\mathbf{M Z}(u)$

Proof. Use Lemma 5 and the characterization of Laraki (2001) [7] or Rosenberg and Sorin (2001) $[15]$.

\subsection{The finitely repeated game}

We now turn to the study of the finitely repeated game: recall that the payoff of the $n$-stage game is given by $\frac{1}{n} \sum_{k=1}^{n} g\left(x_{k}, y_{k}, p, q\right)$ and that $v_{n}$ denotes its value. The recursive formula in this framework is:

$$
v_{n}(p, q)=\max _{x \in \mathbf{X}} \min _{y \in \mathbf{Y}}\left[\frac{1}{n} g(x, y, p, q)+\left(1-\frac{1}{n}\right) \sum_{i, j} x(i) y(j) v_{n-1}(p(i), q(j))\right]=\mathbf{T}\left(\frac{1}{n}, v_{n-1}\right) .
$$

Given an integer $n \geq 1$, let $\Pi$ be the uniform partition of $[0,1]$ with mesh $\frac{1}{n}$ and write simply $W_{n}$ for the associate function $V_{\Pi}$. Hence $W_{n}(1, p, q):=0$ and for $m=0, \ldots, n-1, W_{n}\left(\frac{m}{n}, p, q\right)$ satisfies:

$$
W_{n}\left(\frac{m}{n}, p, q\right)=\max _{x \in \Delta(I)^{K}} \min _{y \in \Delta(J)^{L}}\left[\frac{1}{n} g(x, y, p, q)+\sum_{i, j} x(i) y(j) W_{n}\left(\frac{m+1}{n}, p(i), q(j)\right)\right]
$$

Note that $W_{n}\left(\frac{m}{n}, p, q, \omega\right)=\left(1-\frac{m}{n}\right) v_{n-m}(p, q, \omega)$ and if $W_{n}$ converges uniformly to $W, v_{n}$ converges uniformly to some function $v$, with $W(t, p, q)=(1-t) v(p, q)$.

Let $\mathcal{T}$ be the set of real continuous functions $W$ on $[0,1] \times P \times Q$ such that for all $t \in[0,1], W(t, . ..) \in$ $\mathcal{S} . \mathbf{X}(t, p, q, W)$ is the set of optimal strategies for Player 1 in $\mathbf{T}(0, W(t, .,)$.$) and \mathbf{Y}(t, p, q, W)$ is defined accordingly.

Let $\mathcal{T}_{0}$ be the set of accumulation points of the family $\left\{W_{n}\right\}$ for the uniform convergence.

Lemma $7 \mathcal{T}_{0} \neq \emptyset$ and $\mathcal{T}_{0} \subset \mathcal{T}$.

Proof. $W_{n}(t, .,$.$) is C$-Lipschitz continuous in $(p, q)$ for the $L^{1}$ norm since the payoff, given the strategies $(\sigma, \tau)$ of the players, is of the form $\sum_{k, \ell} p^{k} q^{\ell} A^{k \ell}(\sigma, \tau)$. Using Lemma 1 it follows that the family $\left\{W_{n}\right\}$ is uniformly Lipschitz on $[0,1] \times P \times Q$ hence is relatively compact for the uniform norm. Note finally using $(10)$ that $\mathcal{T}_{0} \subset \mathcal{T}$.

We now define two properties for a function $W \in \mathcal{T}$ and a $C^{1}$ test function $\phi:[0,1] \rightarrow \mathbb{R}$. 
- P1: If $t \in[0,1)$ is such that $\mathbf{X}(t, p, q, W)$ is non-revealing and $W(\cdot, p, q)-\phi(\cdot)$ has a global maximum at $t$, then $u(p, q)+\phi^{\prime}(t) \geq 0$.

- P2: If $t \in[0,1)$ is such that $\mathbf{Y}(t, p, q, W)$ is non-revealing and $W(\cdot, p, q)-\phi(\cdot)$ has a global minimum at $t$ then $u(p, q)+\phi^{\prime}(t) \leq 0$.

Lemma 8 Any $W \in \mathcal{T}_{0}$ satisfies $\mathbf{P 1}$ and $\mathbf{P 2}$.

Note that this result is the variational counterpart of Lemma 2.

Proof. Let $t \in[0,1), p$ and $q$ be such that $\mathbf{X}(t, p, q, W)$ is non-revealing and $W(\cdot, p, q)-\phi(\cdot)$ admits a global maximum at $t$. Adding the function $s \mapsto(s-t)^{2}$ to $\phi$ if necessary, we can assume that this global maximum is strict.

Let $W_{n_{k}}$ be a subsequence converging uniformly to $W$. Put $m=n_{k}$ and define $\theta(m) \in\{0, \ldots, m-$ $1\}$ such that $\frac{\theta(m)}{m}$ is a global maximum of $W_{m}(\cdot, p, q)-\phi(\cdot)$ on the set $\{0, \ldots, m-1\}$. Since $t$ is a strict maximum, one has $\frac{\theta(m)}{m} \rightarrow t$, as $m \rightarrow \infty$. From (11):

$$
W_{m}\left(\frac{\theta(m)}{m}, p, q\right)=\max _{x \in \mathbf{X}} \min _{y \in \mathbf{Y}}\left[\frac{1}{m} g(x, y, p, q)+\sum_{i, j} x(i) y(j) W_{m}\left(\frac{\theta(m)+1}{m}, p(i), q(j)\right)\right] \text {. }
$$

Let $x_{m} \in \mathbf{X}$ be optimal for player 1 in the above formula and let $j \in J$ be any (non-revealing) pure action of player 2 . Then:

$$
W_{m}\left(\frac{\theta(m)}{m}, p, q\right) \leq \frac{1}{m} g\left(x_{m}, j, p, q\right)+\sum_{i} x_{m}(i) W_{m}\left(\frac{\theta(m)+1}{m}, p_{m}(i), q\right) .
$$

By concavity of $W_{m}$ with respect to $p$, we have

$$
\sum_{i \in I} x_{m}(i) W_{m}\left(\frac{\theta(m)+1}{m}, p_{m}(i), q\right) \leq W_{m}\left(\frac{\theta(m)+1}{m}, p, q\right)
$$

hence:

$$
0 \leq g\left(x_{m}, j, p, q\right)+m\left[W_{m}\left(\frac{\theta(m)+1}{m}, p, q\right)-W_{m}\left(\frac{\theta(m)}{m}, p, q\right)\right] .
$$

Since $\frac{\theta(m)}{m}$ is a global maximum of $W_{(m)}(\cdot, p, q)-\phi(\cdot)$ on $\{0, \ldots, m-1\}$ one has:

$$
W_{m}\left(\frac{\theta(m)+1}{m}, p, q\right)-W_{m}\left(\frac{\theta(m)}{m}, p, q\right) \leq \phi\left(\frac{\theta(m)+1}{m}\right)-\phi\left(\frac{\theta(m)}{m}\right)
$$

so that:

$$
0 \leq g\left(x_{m}, j, p, q\right)+m\left[\phi\left(\frac{\theta(m)+1}{m}\right)-\phi\left(\frac{\theta(m)}{m}\right)\right] .
$$

Since $\mathbf{X}$ is compact, one can assume without loss of generality that $\left\{x_{m}\right\}$ converges to some $x$. Note that $x$ belongs to $\mathbf{X}(t, p, q, W)$ by upper semicontinuity using the uniform convergence of $W_{m}$ to $W$. Hence $x$ is non-revealing. Thus, passing to the limit one obtains:

$$
0 \leq g(x, j, p, q)+\phi^{\prime}(t) .
$$

Since this inequality holds true for every $j \in J$, we also have:

$$
\min _{j \in J} g(x, j, p, q)+\phi^{\prime}(t) \geq 0 \text {. }
$$


Taking the maximum with respect to $x \in N R_{\mathbf{X}}(p)$ gives the desired result:

$$
u(p, q)+\phi^{\prime}(t) \geq 0 .
$$

The comparison principle in this case is given by the next result.

Lemma 9 Let $W_{1}$ and $W_{2}$ in $\mathcal{T}$ satisfying $\mathbf{P 1}, \mathbf{P 2}$ and

- P3: $W_{1}(1, p, q) \leq W_{2}(1, p, q)$ for any $(p, q) \in \Delta(K) \times \Delta(L)$.

Then $W_{1} \leq W_{2}$ on $[0,1] \times \Delta(K) \times \Delta(L)$.

Proof. We argue by contradiction, assuming that

$$
\max _{t \in[0,1], p \in P, q \in Q}\left[W_{1}(t, p, q)-W_{2}(t, p, q)\right]=\delta>0 .
$$

Then, for $\varepsilon>0$ sufficiently small,

$$
\delta(\varepsilon):=\max _{t \in[0,1], s \in[0,1], p \in P, q \in Q}\left[W_{1}(t, p, q)-W_{2}(s, p, q)-\frac{(t-s)^{2}}{2 \varepsilon}+\varepsilon s\right]>0 .
$$

Moreover $\delta(\varepsilon) \rightarrow \delta$ as $\varepsilon \rightarrow 0$.

We claim that there is $\left(t_{\varepsilon}, s_{\varepsilon}, p_{\varepsilon}, q_{\varepsilon}\right)$, point of maximum in (12), such that $\mathbf{X}\left(t_{\varepsilon}, p_{\varepsilon}, q_{\varepsilon}, W_{1}\right)$ is non-revealing for player 1 and $\mathbf{Y}\left(s_{\varepsilon}, p_{\varepsilon}, q_{\varepsilon}, W_{2}\right)$ is non-revealing for player 2. The proof of this claim is like Lemma 3 and follows again Mertens Zamir (1971) [11]. Let $\left(t_{\varepsilon}, s_{\varepsilon}, p_{\varepsilon}^{\prime}, q_{\varepsilon}^{\prime}\right)$ be a maximum point of $(12)$ and $C(\varepsilon)$ be the set of maximum points in $P \times Q$ of the function: $(p, q) \mapsto W_{1}\left(t_{\varepsilon}, p, q\right)-W_{2}\left(s_{\varepsilon}, p, q\right)$. This is a compact set. Let $\left(p_{\varepsilon}, q_{\varepsilon}\right)$ be an extreme point of the convex hull of $C(\varepsilon)$. By Caratheodory's theorem, this is also an element of $C(\varepsilon)$. Let $x_{\varepsilon} \in \mathbf{X}\left(t_{\varepsilon}, p_{\varepsilon}, q_{\varepsilon}, W_{1}\right)$ and $y_{\varepsilon} \in \mathbf{Y}\left(s_{\varepsilon}, p_{\varepsilon}, q_{\varepsilon}, W_{2}\right)$. Since $W_{1}$ and $W_{2}$ are in $\mathcal{T}$, we have:

$$
W_{1}\left(t_{\varepsilon}, p_{\varepsilon}, q_{\varepsilon}\right)-W_{2}\left(s_{\varepsilon}, p_{\varepsilon}, q_{\varepsilon}\right) \leq \sum_{i, j} x_{\varepsilon}(i) y_{\varepsilon}(j)\left[W_{1}\left(t_{\varepsilon}, p_{\varepsilon}(i), q_{\varepsilon}(j)\right)-W_{2}\left(s_{\varepsilon}, p_{\varepsilon}(i), q_{\varepsilon}(j)\right)\right] .
$$

By optimality of $\left(p_{\varepsilon}, q_{\varepsilon}\right)$, one deduces that, for every $i$ and $j$ with $x_{\varepsilon}(i)>0$ and $y_{\varepsilon}(j)>0$, $\left(p_{\varepsilon}(i), q_{\varepsilon}(j)\right) \in C(\varepsilon)$. Since $\left(p_{\varepsilon}, q_{\varepsilon}\right)=\sum_{i, j} x_{\varepsilon}(i) y_{\varepsilon}(j)\left(p_{\varepsilon}(i), q_{\varepsilon}(j)\right)$ and $\left(p_{\varepsilon}, q_{\varepsilon}\right)$ is an extreme point of the convex hull of $C(\varepsilon)$ one concludes that $\left(p_{\varepsilon}(i), q_{\varepsilon}(j)\right)=\left(p_{\varepsilon}, q_{\varepsilon}\right)$ for all $i$ and $j: x_{\varepsilon}$ and $y_{\varepsilon}$ are non-revealing. Therefore we have constructed $\left(t_{\varepsilon}, s_{\varepsilon}, p_{\varepsilon}, q_{\varepsilon}\right)$ as claimed.

Finally we note that $t_{\varepsilon}<1$ and $s_{\varepsilon}<1$ for $\varepsilon$ sufficiently small, because $\delta(\varepsilon)>0$ and $W_{1}(1, p, q) \leq$ $W_{2}(1, p, q)$ for any $(p, q) \in P \times Q$ by $\mathbf{P 3}$.

Since the map $t \mapsto W_{1}\left(t, p_{\varepsilon}, q_{\varepsilon}\right)-\frac{\left(t-s_{\varepsilon}\right)^{2}}{2 \varepsilon}$ has a global maximum at $t_{\varepsilon}$ and since $\mathbf{X}\left(t_{\varepsilon}, p_{\varepsilon}, q_{\varepsilon}, W_{1}\right)$ is non-revealing for player 1 , condition $\mathbf{P} \mathbf{1}$ implies that

$$
u\left(p_{\varepsilon}, q_{\varepsilon}\right)+\frac{t_{\varepsilon}-s_{\varepsilon}}{\varepsilon} \geq 0 .
$$

In the same way, since the map $s \mapsto W_{2}\left(s, p_{\varepsilon}, q_{\varepsilon}\right)+\frac{\left(t_{\varepsilon}-s\right)^{2}}{2 \varepsilon}-\varepsilon s$ has a global minimum at $s_{\varepsilon}$ and since $\mathbf{Y}\left(s_{\varepsilon}, p_{\varepsilon}, q_{\varepsilon}, W_{2}\right)$ is non-revealing for player 2, we have by condition $\mathbf{P 2}$ that

$$
u\left(p_{\varepsilon}, q_{\varepsilon}\right)+\frac{t_{\varepsilon}-s_{\varepsilon}}{\varepsilon}+\varepsilon \leq 0 .
$$

This latter inequality contradicts (13).

We are now ready to prove the convergence result for $\lim _{n \rightarrow \infty} v_{n}$. 
Proposition $10 W_{n}$ converges uniformly to the unique point $W \in \mathcal{T}$ that satisfies the variational inequalities $\mathbf{P} 1$ and $\mathbf{P 2}$ and the terminal condition $W(0, p, q)=0$.

Consequently, $v_{n}(p, q)$ converges uniformly to $v(p, q)=W(0, p, q)$ and $W(t, p, q)=(1-t) v(p, q)$, where $v=\mathbf{M Z}(u)$.

Proof. Let $W \in \mathcal{T}_{0}$. From Lemma $8, W$ satisfies the variational inequalities $\mathbf{P 1}$ and $\mathbf{P 2}$. Moreover, $W(1, p, q)=0$. Since, from Lemma 9 , there is at most one function fulfilling these conditions, we obtain convergence of the family $\left\{W_{n}\right\}$.

Consequently, $v_{n}(p, q)$ converges uniformly to $v(p, q)=W(0, p, q)$ and $W(t, p, q)=(1-t) v(p, q)$. In particular if one considers $\phi(t)=W(t, p, q)$ as test function, then $\phi^{\prime}(t)=-v(p, q)$. Now P1 and $\mathbf{P 2}$ reduce to Lemma 2 hence via Lemma 5 to the variational characterization of $\mathbf{M Z}(u)$.

\subsection{General evaluation}

Consider now an arbitrarily evaluation probability $\mu$ on $\mathbb{I}^{*}$, with $\mu_{n} \geq \mu_{n+1}$, inducing a partition $\Pi$. Let $V_{\Pi}\left(t_{k}, p, q\right)$ be the value of the game starting at time $t_{k}$. One has $V_{\Pi}(1, p, q):=0$ and

$$
V_{\Pi}\left(t_{n}, p, q\right)=\max _{x \in \mathbf{X}} \min _{y \in \mathbf{Y}}\left[\mu_{n+1} g(x, y, p, q)+\sum_{i, j} x(i) y(j) V_{\Pi}\left(t_{n+1}, p(i), q(j)\right)\right] .
$$

Moreover $V_{\Pi}$ belongs to $\mathcal{F}$ and is $C$ Lipschitz in $(p, q)$.

Lemma 1 then implies that any family of values $V_{\Pi(m)}$ associated to partitions $\Pi(m)$ with $\mu_{1}(m) \rightarrow 0$ as $m \rightarrow \infty$ has an accumulation point. Denote by $\mathcal{T}_{1}$ the set of those functions. Then $\mathcal{T}_{1} \subset \mathcal{T}$ by (14) and lemma 8 extends in a natural way: let $\bar{V} \in \mathcal{T}_{1}$ and $V_{\Pi(m)} \rightarrow \bar{V}$ uniformly. Let $t_{n}^{m}$ be a global maximum of $V_{\Pi(m)}(., p, q)-\phi($.$) on \Pi(m)$. Then $t_{n}^{m} \rightarrow t$ and one has

$$
0 \leq g\left(x_{n}, j, p, q\right)+\frac{1}{\mu_{n}(m)}\left[V_{\Pi(m)}\left(t_{n+1}^{m}, p, q\right)-V_{\Pi(m)}\left(t_{n}^{m}, p, q\right)\right]
$$

hence

$$
0 \leq g\left(x_{n}, j, p, q\right)+\frac{1}{\mu_{n}(m)}\left[\phi\left(t_{n+1}^{m}\right)-\phi\left(t_{n}^{m}\right)\right]
$$

and letting $n \rightarrow \infty$ the result follows.

Using Lemma 9 this implies the convergence. Thus:

Proposition $11 V_{\Pi(m)}$ converges uniformly to the unique point $V \in \mathcal{T}$ that satisfies the variational inequalities $\mathbf{P} \mathbf{1}$ and $\mathbf{P} \mathbf{2}$ and the terminal condition $V(0, p, q)=0$.

Consequently, $v_{\Pi(m)}(p, q)$ converges uniformly to $v(p, q)=V(0, p, q)$ and $V(t, p, q)=(1-t) v(p, q)$. Moreover $v=\mathbf{M Z}(u)$.

In particular the convergence of $\left\{V_{\Pi(m)}\right\}$ to the same limit for any family of decreasing partitions allows to use $\lim _{\lambda \rightarrow 0} v_{\lambda}$ to characterize the limit.

\section{Splitting games}

We consider now the framework of splitting games, Sorin (2002) [20], p. 78. Let $P$ and $Q$ be two simplexes (or product of simplexes) of some finite dimensional spaces, and $H$ a $C$-Lipschitz function from $P \times Q$ to $\mathbb{R}$. The corresponding Shapley operator is defined on continuous saddle (concave-convex) real functions $f$ on $P \times Q$ by

$$
\mathbf{T}(\lambda, f)(p, q)=\operatorname{val}_{\mu \in M_{p}^{P} \times \nu \in M_{q}^{Q}} \int_{P \times Q}\left[\left(\lambda H\left(p^{\prime}, q^{\prime}\right)+(1-\lambda) f\left(p^{\prime}, q^{\prime}\right)\right] \mu\left(d p^{\prime}\right) \nu\left(d q^{\prime}\right)\right.
$$


where $M_{p}^{P}$ stands for the set of Borel probabilities on $P$ with expectation $p$ (and similarly for $\left.M_{q}^{Q}\right)$.

The associated repeated game is played as follows: at stage $n+1$ knowing the state $\left(p_{n}, q_{n}\right)$ player 1 (resp. player 2) chooses $\mu_{n+1} \in M_{p_{n}}^{P}$ (resp. $\nu \in M_{q_{n}}^{Q}$ ). A new state $\left(p_{n+1}, q_{n+1}\right)$ is selected according to these distributions and the stage payoff is $H\left(p_{n+1}, q_{n+1}\right)$. We denote by $V_{\lambda}$ the value of the discounted game and by $v_{n}$ the value of the $n$-stage game.

A procedure analogous to the previous study of discounted games with incomplete information has been developed by Laraki [6], [7], [9].

\subsection{The discounted game}

The next properties are established in Laraki (2001) [7].

Let $\mathcal{G}$ be the set of C-Lipschitz saddle functions on $P \times Q$.

Lemma 12 The Shapley operator $\mathbf{T}(\lambda, \cdot)$ maps $\mathcal{G}$ to itself and $V_{\lambda}(p, q)$ is the only fixed point of $T(\lambda,$.$) in \mathcal{G}$.

The corresponding projective operator is the splitting operator $\Psi$ :

$$
\Psi(f)(p, q)=\operatorname{val}_{M_{p}^{P} \times \nu M_{q}^{Q}} \int_{P \times Q} f\left(p^{\prime}, q^{\prime}\right) \mu\left(d p^{\prime}\right) \nu\left(d q^{\prime}\right)
$$

and we denote again by $\mathcal{S}$ its set of fixed points. Given $W \in \mathcal{S}, \mathbf{P}(p, q, W) \subset M_{p}^{P}$ denotes the set of optimal strategies of player 1 in (15) for $\Psi(W)(p, q)$. We say that $\mathbf{P}(p, q, W)$ is nonrevealing if it is reduced to $\delta_{p}$, the Dirac mass at $p$. We use the symmetric notation $\mathbf{Q}(p, q, W)$ and terminology for player 2 .

We define two properties for functions in $\mathcal{S}$.

- A1: If $\mathbf{P}(p, q, W)$ is non-revealing, then $W(p, q) \leq H(p, q)$.

- A2: If $\mathbf{Q}(p, q, W)$ is non-revealing, then $W(p, q) \geq H(p, q)$.

Proposition $13 V_{\lambda}$ converges uniformly to the unique point $V \in \mathcal{S}$ that satisfies the variational inequalities A1 and A2.

The link with the $\mathbf{M Z}$ operator is as follows: as in Lemma 5 one defines:

- B1: If $p \in \mathcal{E} W(., q)$, then $W(p, q) \leq H(p, q)$.

- B2: If $q \in \mathcal{E} W(p,$.$) , then W(p, q) \geq H(p, q)$

(where, as before, $\mathcal{E} V$ denotes the set of extreme points of a convex or concave map $V$ ). Then one has $\mathbf{A i}$ implies $\mathbf{B i}, \mathbf{i}=\mathbf{1}, \mathbf{2}$ and

Proposition 14 Let $G \in \mathcal{G}$. Then $G$ satisfies $\mathbf{B} 1$ and $\mathbf{B 2}$ iff $G=\mathbf{M Z}(H)$.

\subsection{The finitely repeated game}

Recall the recursive formula defining by induction the value of the $n$ stage game $v_{n} \in \mathcal{G}$, using Lemma 12:

$$
v_{n}(p, q)=\operatorname{val}_{M_{p}^{P} \times M_{q}^{Q}} \int_{P \times Q}\left[\frac{1}{n} H\left(p^{\prime}, q^{\prime}\right)+\left(1-\frac{1}{n}\right) v_{n-1}\left(p^{\prime}, q^{\prime}\right)\right] \mu\left(d p^{\prime}\right) \nu\left(d q^{\prime}\right)=\mathbf{T}\left(\frac{1}{n}, V_{n-1}\right) .
$$


For each integer $n \geq 1$, let $W_{n}(1, p, q):=0$ and for $m=0, \ldots, n-1$ define $W_{n}\left(\frac{m}{n}, p, q\right)$ inductively as follows:

$$
W_{n}\left(\frac{m}{n}, p, q\right)=\operatorname{val}_{M_{p}^{P} \times M_{q}^{Q}} \int_{P \times Q}\left[\frac{1}{n} H\left(p^{\prime}, q^{\prime}\right)+W_{n}\left(\frac{m+1}{n}, p^{\prime}, q^{\prime}\right)\right] \mu\left(d p^{\prime}\right) \nu\left(d q^{\prime}\right) .
$$

By induction we have $W_{n}\left(\frac{m}{n}, p, q\right)=\left(1-\frac{m}{n}\right) v_{n-m}(p, q)$. Note that $W_{n}$ is the function on $[0,1] \times$ $P \times Q$ associated to the uniform partition of mesh $\frac{1}{n}$.

Lemma $15 W_{n}$ is Lipschitz continuous uniformly in $n$ on $\left\{\frac{m}{n}, m \in\{0, \ldots, n\}\right\} \times P \times Q$.

Proof. By Lemma $12 W_{n}(t, .,$.$) belongs to \mathcal{G}$ for any $t$. As for Lipschitz continuity with respect to $t$, we have, if $\mu$ is optimal in (17) and by Jensen's inequality:

$$
\begin{aligned}
W_{n}\left(\frac{m}{n}, p, q\right) & \leq \int_{P \times Q} \frac{1}{n} H\left(p^{\prime}, q\right)+W_{n}\left(\frac{m+1}{n}, p^{\prime}, q\right) d \mu\left(p^{\prime}\right) \\
& \leq \frac{\|H\|_{\infty}}{n}+W_{n}\left(\frac{m+1}{n}, p, q\right) .
\end{aligned}
$$

One gets the reverse inequality $W_{n}\left(\frac{m}{n}, p, q\right) \geq-\frac{\|H\|_{\infty}}{n}+W_{n}\left(\frac{m+1}{n}, p, q\right)$ with the symmetric arguments. Therefore $W_{n}(\cdot, p, q)$ is $\|H\|_{\infty}$-Lipschitz continuous.

Let $\mathcal{T}$ be the set of real continuous functions $W$ on $[0,1] \times P \times Q$ such that for all $t \in$ $[0,1], W(t, . ..) \in \mathcal{S} . \mathbf{P}(t, p, q, W)$ is defined as $\mathbf{P}(p, q, W(t, .,)$.$) and \mathbf{Q}(t, p, q, W)$ as $\mathbf{Q}(p, q, W(t, . .)$.$) .$ Let $\mathcal{T}_{0}$ be the set of accumulation points of the family $W_{n}$. Using (17), we have that $\mathcal{T}_{0} \subset \mathcal{T}$. We introduce two properties for a function $W \in \mathcal{T}$ and any $C^{1}$ test function $\phi:[0,1] \rightarrow \mathbb{R}$.

- PS1: If, for some $t \in[0,1), \mathbf{P}(t, p, q, W)$ is non-revealing and $W(\cdot, p, q)-\phi(\cdot)$ has a global maximum at $t$, then $H(p, q)+\phi^{\prime}(t) \geq 0$.

- PS2: If, for some $t \in[0,1), \mathbf{Q}(t, p, q, W)$ is non-revealing and $W(\cdot, p, q)-\phi(\cdot)$ has a global minimum at $t$ then $H(p, q)+\phi^{\prime}(t) \leq 0$.

Lemma 16 Any $W \in \mathcal{T}_{0}$ satisfies PS1 and PS2.

Proof. The proof is very similar to the proof of Lemma 8.

Let $t \in[0,1), p$ and $q$ be such that $\mathbf{P}(t, p, q, W)$ is non-revealing and $W(\cdot, p, q)-\phi(\cdot)$ admits a global maximum at $t$. Adding $(\cdot-t)^{2}$ to $\phi$ if necessary, we can assume that this global maximum is strict.

Let $W_{n_{k}}$ be a sequence converging uniformly to $W$. Write $m=n_{k}$ and define $\theta(m) \in\{0, \ldots, m-1\}$ such that $\frac{\theta(m)}{m}$ is a global maximum of $W_{m}(\cdot, p, q)-\phi(\cdot)$ on $\{0, \ldots, m-1\}$. Since $t$ is a strict maximum, we have $\frac{\theta(m)}{m} \rightarrow t$. By (17) we have that:

$$
W_{m}\left(\frac{\theta(m)}{m}, p, q\right)=\operatorname{val}_{M_{p}^{P} \times M_{q}^{Q}} \int_{P \times Q}\left[\frac{1}{m} H\left(p^{\prime}, q^{\prime}\right)+W_{m}\left(\frac{\theta(m)+1}{m}, p^{\prime}, q^{\prime}\right)\right] \mu\left(d p^{\prime}\right) \nu\left(d q^{\prime}\right) .
$$

Let $\mu_{m}$ be optimal for player 1 in the above formula and let $\nu=\delta_{q}$ be the Dirac mass at $q$. Then:

$$
W_{m}\left(\frac{\theta(m)}{m}, p, q\right) \leq \int_{P} \frac{1}{m} H\left(p^{\prime}, q\right) \mu_{m}\left(d p^{\prime}\right)+\int_{P} W_{m}\left(\frac{\theta(m)+1}{m}, p^{\prime}, q\right) \mu_{m}\left(d p^{\prime}\right) .
$$

By concavity of $W_{m}$ with respect to $p$, we have

$$
\int_{P} W_{m}\left(\frac{\theta(m)+1}{m}, p^{\prime}, q\right) \mu_{m}\left(d p^{\prime}\right) \leq W_{m}\left(\frac{\theta(m)+1}{m}, p, q\right)
$$


Hence:

$$
0 \leq \int_{P} H\left(p^{\prime}, q\right) \mu_{m}\left(d p^{\prime}\right)+m\left[W_{m}\left(\frac{\theta(m)+1}{m}, p, q\right)-W_{m}\left(\frac{\theta(m)}{m}, p, q\right)\right] .
$$

Since $\frac{\theta(m)}{m}$ is a global maximum of $W_{m}(\cdot, p, q)-\phi(\cdot)$ on $\{0, \ldots, m-1\}$ one has:

$$
W_{m}\left(\frac{\theta(m)+1}{m}, p, q\right)-W_{m}\left(\frac{\theta(m)}{m}, p, q\right) \leq \phi\left(\frac{\theta(m)+1}{m}\right)-\phi\left(\frac{\theta(m)}{m}\right)
$$

So that

$$
0 \leq \int_{P} H\left(p^{\prime}, q\right) \mu_{m}\left(d p^{\prime}\right)+m\left[\phi\left(\frac{\theta(m)+1}{m}\right)-\phi\left(\frac{\theta(m)}{m}\right)\right]
$$

Since $M_{p}^{P}$ is compact, one can assume without loss of generality that $\left\{\mu_{m}\right\}$ converges to some $\mu$. Note that $\mu$ belongs to $\mathbf{P}(t, p, q, W)$ by upper semicontinuity and uniform convergence of $W_{m}$ to $W$. Hence $\mu$ is non-revealing: $\mu=\delta_{p}$. Thus, passing to the limit in (18) one obtains:

$$
0 \leq H(p, q)+\phi^{\prime}(t)
$$

The comparison principle in this case is given by the next result.

Lemma 17 Let $W_{1}$ and $W_{2}$ in $\mathcal{T}$ satisfying PS1, PS2 and

- PS3: $W_{1}(1, p, q) \leq W_{2}(1, p, q)$ for any $(p, q) \in \Delta(K) \times \Delta(L)$.

Then $W_{1} \leq W_{2}$ on $[0,1] \times \Delta(K) \times \Delta(L)$.

The proof is exactly similar to the proof of Lemma 9.

We are now ready to prove the convergence result for $\lim _{n \rightarrow \infty} v_{n}$ :

Proposition $18 W_{n}$ converges uniformly to the unique point $W \in \mathcal{T}$ that satisfies the variational inequalities PS1 and PS2 and the terminal condition $W(1, p, q)=0$.

Consequently, $v_{n}(p, q)$ converges uniformly to $v(p, q)=W(0, p, q)$ and $W(t, p, q)=(1-t) v(p, q)$. Moreover $v=\mathbf{M Z}(H)$.

Proof. Let $W$ be any limit point of the relatively compact family $W_{n}$. Then, from Lemma 16, $W \in \mathcal{T}_{0}$ satisfies the variational inequalities PS1 and PS2. Moreover, $W(1, p, q)=0$. Since, from Lemma 17, there is at most one map fulfilling these conditions, we obtain convergence.

Consequently, $v_{n}(p, q)$ converges uniformly to $V(p, q)=W(0, p, q)$ and $W(t, p, q)=(1-t) V(p, q)$. In particular if one choose as test function $\phi(t)=W(t, p, q)$, then $\phi^{\prime}(t)=-V(p, q)$, so that PS1 and $\mathbf{P S 2}$ reduce to $\mathbf{A} \mathbf{1}$ and $\mathbf{A 2}$. On concludes by using the variational characterization of $\mathbf{M Z}(u)$ in Proposition 14.

\subsection{General evaluation}

The same results extend to the general evaluation case defined by a partition $\Pi$ with $\mu_{n}$ decreasing. The existence of $V_{\Pi}$ is obtained in two steps. We first let $V_{\Pi}^{n}$ to be 0 on $\left[t_{n}, 1\right]$ and define inductively $V_{\Pi}^{n}\left(t_{m}, . ..\right)$ for $m<n$ by

$$
V_{\Pi}^{n}\left(t_{m}, p, q\right)=\operatorname{val}_{M_{p}^{P} \times M_{q}^{Q}} \int_{P \times Q}\left[\mu_{m+1} H\left(p^{\prime}, q^{\prime}\right)+V_{\Pi}^{n}\left(t_{m+1}, p^{\prime}, q^{\prime}\right)\right] \mu\left(d p^{\prime}\right) \nu\left(d q^{\prime}\right) .
$$

It follows that $V_{\Pi}^{n} \in \mathcal{G}$ by Lemma 12 and converges uniformly to $V_{\Pi}$. Then the proof follows exactly the same steps than in Part 2. 


\subsection{Time dependent case}

We consider here the case where the function $H$ may depend on the stage.

To be able to study the asymptotic behavior one has to define $H$ directly in the limit game: the map $H$ is a continuous real function on $[0,1] \times P \times Q$.

For each integer $n$, let $Z_{n}(1, p, q):=0$ and for $m=0, \ldots, n-1$ define $Z_{n}\left(\frac{m}{n}, p, q\right)$ inductively as follows:

$$
Z_{n}\left(\frac{m}{n}, p, q\right)=\operatorname{val}_{M_{p}^{P} \times M_{q}^{Q}} \int_{P \times Q}\left[\frac{1}{n} H\left(\frac{m}{n}, p^{\prime}, q^{\prime}\right)+Z_{n}\left(\frac{m+1}{n}, p^{\prime}, q^{\prime}\right)\right] \mu\left(d p^{\prime}\right) \nu\left(d q^{\prime}\right) .
$$

By induction each function $Z_{n}\left(\frac{m}{n}, . ..\right)$ is in $\mathcal{G}$ and one can show as in Lemma 15 that $Z_{n}$ is uniformly Lipschitz continuous on $\left\{\frac{m}{n}, m \in\{0, \ldots, n\}\right\} \times P \times Q$.

Remark : An alternative choice is to replace $\frac{1}{n} H\left(\frac{m}{n}, p^{\prime}, q^{\prime}\right)$ by $\int_{\frac{m}{n}}^{\frac{m+1}{n}} H\left(t, p^{\prime}, q^{\prime}\right) d t$.

Note that the projective operator is the same than in the autonomous case. Let $\mathcal{T}$ be the set of real functions $Z$ on $[0,1] \times P \times Q$ such that for all $t \in[0,1], Z(t, . ..) \in \mathcal{S}$. We define $\mathbf{P}(t, p, q, Z)$ and $\mathbf{Q}(t, p, q, Z)$ as before and denote by $\mathcal{Z}_{0}$ the set of accumulation points of the family $Z_{n}$. We note that $\mathcal{Z}_{0} \subset \mathcal{T}$.

We define two properties for a function $Z \in \mathcal{T}$ and all $C^{1}$ test function $\phi:[0,1] \rightarrow \mathbb{R}$.

- PST1: If, for some $t \in[0,1), \mathbf{P}(t, p, q, Z)$ is non-revealing and $Z(\cdot, p, q)-\phi(\cdot)$ has a global maximum at $t$, then $H(t, p, q)+\phi^{\prime}(t) \geq 0$.

- PST2: If, for some $t \in[0,1), \mathbf{Q}(t, p, q, Z)$ is non-revealing and $Z(\cdot, p, q)-\phi(\cdot)$ has a global minimum at $t$ then $H(t, p, q)+\phi^{\prime}(t) \leq 0$.

Lemma 19 Any $Z \in \mathcal{Z}_{0}$ satisfies PST1 and PST2.

Proof. Let $t \in[0,1), p$ and $q$ be such that $\mathbf{P}(t, p, q, Z)$ is non-revealing and $Z(\cdot, p, q)-\phi(\cdot)$ admits a global maximum at $t$. Adding $(\cdot-t)^{2}$ to $\phi$ if necessary, we can assume that this global maximum is strict.

Let $Z_{n_{k}}$ be a sequence converging uniformly to $Z$. Write $m=n_{k}$ and define $\theta(m) \in\{0, \ldots, m-1\}$ such that $\frac{\theta(m)}{m}$ is a global maximum of $Z_{m}(\cdot, p, q)-\phi(\cdot)$ on $\{0, \ldots, m-1\} . t$ being a strict maximum $\frac{\theta(m)}{m} \rightarrow t$. By (20) we have that:

$$
Z_{m}\left(\frac{\theta(m)}{m}, p, q\right)=\sup _{\mu \in M_{p}^{P}} \inf _{\nu \in M_{q}^{Q}} \int_{P \times Q}\left[\frac{1}{m} H\left(\frac{\theta(m)}{m}, p^{\prime}, q^{\prime}\right)+Z_{m}\left(\frac{\theta(m)+1}{m}, p^{\prime}, q^{\prime}\right)\right] \mu\left(d p^{\prime}\right) \mu\left(d q^{\prime}\right) .
$$

Let $\mu_{m}$ be optimal for player I in the above formula and let $\nu=\delta_{q}$ be the Dirac mass at $q$. Then:

$$
Z_{m}\left(\frac{\theta(m)}{m}, p, q\right) \leq \int_{P} \frac{1}{m} H\left(\frac{\theta(m)}{m}, p^{\prime}, q^{\prime}\right) \mu_{m}\left(d p^{\prime}\right)+\int_{P} Z_{n}\left(\frac{\theta(m)+1}{m}, p^{\prime}, q\right) \mu_{m}\left(d p^{\prime}\right) .
$$

By concavity of $Z_{m}$ with respect to $p$, we have

$$
\int_{P} Z_{m}\left(\frac{\theta(m)+1}{m}, p^{\prime}, q\right) \mu_{m}\left(d p^{\prime}\right) \leq Z_{m}\left(\frac{\theta(m)+1}{m}, p, q\right) .
$$

Hence:

$$
0 \leq \int_{P} H\left(\frac{\theta(m)}{m}, p^{\prime}, q^{\prime}\right) \mu_{m}\left(d p^{\prime}\right)+m\left[Z_{m}\left(\frac{\theta(m)+1}{m}, p, q\right)-Z_{m}\left(\frac{\theta(m)}{m}, p, q\right)\right] .
$$


Since $\frac{\theta(m)}{m}$ is a global maximum of $Z_{\varphi(m)}(\cdot, p, q)-\phi(\cdot)$ on $\{0, \ldots, m-1\}$ one has:

$$
Z_{m}\left(\frac{\theta(m)+1}{m}, p, q\right)-Z_{m}\left(\frac{\theta(m)}{m}, p, q\right) \leq \phi\left(\frac{\theta(m)+1}{m}\right)-\phi\left(\frac{\theta(m)}{m}\right) .
$$

$M_{p}^{P}$ being compact, one can assume without loss of generality that $\left\{\mu_{m}\right\}$ converges to some $\mu$. Note that $\mu$ belongs to $\mathbf{P}(t, p, q, Z)$ by upper semicontinuity and uniform convergence of $Z_{n}$ to $Z$. Hence $\mu=\delta_{p}$ is non-revealing. Thus, passing to the limit one obtains:

$$
0 \leq H(t, p, q)+\phi^{\prime}(t) \text {. }
$$

The comparison principle in this case is given by the next result.

Lemma 20 Let $Z_{1}$ and $Z_{2}$ in $\mathcal{T}$ satisfying PS1, PS2 and

- PS3: $Z_{1}(1, p, q) \leq Z_{2}(1, p, q)$ for any $(p, q) \in \Delta(K) \times \Delta(L)$.

Then $Z_{1} \leq Z_{2}$ on $[0,1] \times \Delta(K) \times \Delta(L)$.

Proof. We argue by contradiction, assuming that, for some $\gamma>0$ small,

$$
\max _{t \in[0,1], p \in P, q \in Q}\left[Z_{1}(t, p, q)-Z_{2}(t, p, q)-\gamma(1-t)\right]=\delta>0 .
$$

Then, for $\varepsilon>0$ sufficiently small,

$$
\delta(\varepsilon):=\max _{t \in[0,1], s \in[0,1], p \in P, q \in Q}\left[Z_{1}(t, p, q)-Z_{2}(s, p, q)-\frac{(t-s)^{2}}{2 \varepsilon}+-\gamma(1-s)\right]>0 .
$$

Moreover $\delta(\varepsilon) \rightarrow \delta$ as $\varepsilon \rightarrow 0$.

Hence as before there is $\left(t_{\varepsilon}, s_{\varepsilon}, p_{\varepsilon}, q_{\varepsilon}\right)$, point of maximum in (12), such that $\mathbf{P}\left(t_{\varepsilon}, p_{\varepsilon}, q_{\varepsilon}, W_{1}\right)$ is non-revealing for player I and $\mathbf{Q}\left(s_{\varepsilon}, p_{\varepsilon}, q_{\varepsilon}, W_{2}\right)$ is non-revealing for player $\mathrm{J}$.

Finally we note that $t_{\varepsilon}<1$ and $s_{\varepsilon}<1$ for $\varepsilon$ sufficiently small, because $\delta(\varepsilon)>0$ and $Z_{1}(1, p, q) \leq$ $Z_{2}(1, p, q)$ for any $p, q$ by $\mathbf{P 3}$.

Since the map $t \mapsto Z_{1}\left(t, p_{\varepsilon}, q_{\varepsilon}\right)-\frac{\left(t-s_{\varepsilon}\right)^{2}}{2 \varepsilon}$ has a global maximum at $t_{\varepsilon}$ and since $\mathbf{P}\left(t_{\varepsilon}, p_{\varepsilon}, q_{\varepsilon}, W_{1}\right)$ is non-revealing for player I, condition PST1 implies that

$$
H\left(t_{\varepsilon}, p_{\varepsilon}, q_{\varepsilon}\right)+\frac{t_{\varepsilon}-s_{\varepsilon}}{\varepsilon} \geq 0
$$

In the same way, since the map $s \mapsto W_{2}\left(s, p_{\varepsilon}, q_{\varepsilon}\right)+\frac{\left(t_{\varepsilon}-s\right)^{2}}{2 \varepsilon}+\gamma(1-s)$ has a global minimum at $s_{\varepsilon}$ and since $\mathbf{Q}\left(s_{\varepsilon}, p_{\varepsilon}, q_{\varepsilon}, W_{2}\right)$ is non-revealing for player J, we have by condition PST2 that

$$
H\left(s_{\varepsilon}, p_{\varepsilon}, q_{\varepsilon}\right)+\frac{t_{\varepsilon}-s_{\varepsilon}}{\varepsilon}+\gamma \leq 0 .
$$

Combining (22) with the previous inequality implies that

$$
H\left(s_{\varepsilon}, p_{\varepsilon}, q_{\varepsilon}\right)-H\left(t_{\varepsilon}, p_{\varepsilon}, q_{\varepsilon}\right)+\gamma \leq 0 .
$$

Letting $\varepsilon \rightarrow 0$, we get a contradiction because $s_{\varepsilon}$ and $t_{\varepsilon}$ converge (up to some subsequence) to the same limit $\bar{t}$.

We are now ready to prove the convergence result for $Z_{n}$.

Proposition $21 Z_{n}$ converges uniformly to the unique point $Z \in \mathcal{T}$ that satisfies the variational inequalities PST1 and PST2 and the terminal condition $Z(1, p, q)=0$.

Remark: the same result obviously holds for any sequence of decreasing evaluation.

Proof. Let $Z$ be any limit point of the relatively compact family $Z_{n}$. Then, from Lemma 19 , $W \in \mathcal{T}_{0}$ satisfies the variational inequalities PST1 and PST2. Moreover, $Z(1, p, q)=0$. Since, from Lemma 20, there is at most one map fulfilling these conditions, we obtain convergence. 


\section{Absorbing games}

An absorbing game is a stochastic game where only one state is non absorbing. In the other states one can assume that the payoff is constant (equal to the value) thus the game is defined by the following elements: two finite sets $I$ and $J$, two (payoff) functions $f, g$ from $I \times J$ to $[-1,1]$ and a function $\pi$ from $I \times J$ to $[0,1]$.

The repeated game with absorbing states is played in discrete time as usual. At stage $m=1,2, \ldots$ (if absorbtion has not yet occurred) player 1 chooses $i_{m} \in I$ and, simultaneously, player 2 chooses $j_{m} \in J:$

(i) the payoff at stage $m$ is $f\left(i_{m}, j_{m}\right)$

(ii) with probability $1-\pi\left(i_{m}, j_{m}\right)$ absorbtion is reached and the payoff in all future stages $n>m$ is $g\left(i_{m}, j_{m}\right)$ and

(iii) with probability $\pi\left(i_{m}, j_{m}\right)$ the situation is repeated at stage $m+1$.

Recall that the asymptotic analysis for these games is due to Kohlberg (1974) [5] who also proved the existence of a uniform value in case of standard signaling.

\subsection{The discounted game}

While the spirit of the proof is the same in the general case we first present the discounted case where the argument is more transparent.

Define $\pi^{*}(i, j)=1-\pi(i, j), f^{*}(i, j)=\pi^{*}(i, j) \times g(i, j)$ and extend bilinearly any $\varphi: I \times J \rightarrow \mathbf{R}$ to $\mathbf{R}^{I} \times \mathbf{R}^{J}$ as follows: $\varphi(\alpha, \beta)=\sum_{i \in I, j \in J} \alpha^{i} \beta^{j} \varphi(i, j)$.

Theorem 22 As $\lambda \rightarrow 0, v_{\lambda}$ converges to $v$ given by

$$
v=\operatorname{val}_{((x, \alpha),(y, \beta)) \in\left(\Delta(I) \times \mathbf{R}_{+}^{I}\right) \times\left(\Delta(J) \times \mathbf{R}_{+}^{J}\right)} \frac{f(x, y)+f^{*}(\alpha, y)+f^{*}(x, \beta)}{1+\pi^{*}(\alpha, y)+\pi^{*}(x, \beta)} .
$$

Remark : The existence of a value is a part of the Theorem. This formula is simpler than the one established in Laraki [10].

Proof. Consider $v_{1}$ an accumulation point of the family $\left\{v_{\lambda}\right\}$ and let $v_{\lambda_{n}}$ converges to $v_{1}$.

We will show that

$$
v_{1} \leq \sup _{(x, \alpha) \in \Delta(I) \times \mathbf{R}_{+}^{I}} \inf _{(y, \beta) \in \Delta(J) \times \mathbf{R}_{+}^{J}} \frac{f(x, y)+f^{*}(\alpha, y)+f^{*}(x, \beta)}{1+\pi^{*}(\alpha, y)+\pi^{*}(x, \beta)} .
$$

A dual argument proves at the same time that the family $\left\{v_{\lambda}\right\}$ converges and that the auxiliary game has a value.

Let $r_{\lambda}(x, y)$ be the discounted payoff induced by a pair of stationary strategies $(x, y) \in \Delta(I) \times$ $\Delta(J)$. Then

$$
r_{\lambda}(x, y)=\frac{\lambda f(x, y)+(1-\lambda) f^{*}(x, y)}{\lambda+(1-\lambda) \pi^{*}(x, y)} .
$$

In particular for any $x_{\lambda}$ optimal for Player 1 one obtains:

$$
v_{\lambda} \leq \frac{\lambda f\left(x_{\lambda}, j\right)+(1-\lambda) f^{*}\left(x_{\lambda}, j\right)}{\lambda+(1-\lambda) \pi^{*}\left(x_{\lambda}, j\right)}, \quad \forall j \in J
$$

that one can write

$$
v_{\lambda} \leq \frac{f\left(x_{\lambda}, j\right)+f^{*}\left(\frac{(1-\lambda) x_{\lambda}}{\lambda}, j\right)}{1+\pi^{*}\left(\frac{(1-\lambda) x_{\lambda}}{\lambda}, j\right)}=c_{j}(\lambda), \quad \forall j \in J .
$$

Note that the ratio $f^{*}\left(\frac{(1-\lambda) x_{\lambda}}{\lambda}, j\right) / \pi^{*}\left(\frac{(1-\lambda) x_{\lambda}}{\lambda}, j\right)$ is bounded, hence $c_{j}(\lambda)$ too. Thus any accumulation point of $c_{j}\left(\lambda_{n}\right)$ is greater than $v_{1}$. Hence by taking an appropriate subsequence in (26) for 
each $j \in J$, we obtain :

$\exists \bar{x} \in \Delta(I)$ accumulation point of $\left\{x_{\lambda_{n}}\right\}$ s.t. $\forall \varepsilon>0, \exists \bar{\alpha}=\frac{(1-\bar{\lambda}) x_{\bar{\lambda}}}{\bar{\lambda}} \in \mathbf{R}_{+}^{I}$ such that

$$
v_{1} \leq \frac{f(\bar{x}, j)+f^{*}(\bar{\alpha}, j)}{1+\pi^{*}(\bar{\alpha}, j)}+\varepsilon, \quad \forall j \in J .
$$

Note that by linearity the same inequality holds for any $y \in \Delta(J)$.

On the other hand, $v_{1}$ is a fixed point of the projective operator and $\bar{x}$ is optimal there, hence

$$
v_{1} \leq \pi(\bar{x}, y) v+f^{*}(\bar{x}, y), \quad \forall y \in \Delta(J) .
$$

Inequality (28) is linear thus extends to

$$
\pi^{*}(\bar{x}, \beta) v_{1} \leq f^{*}(\bar{x}, \beta), \quad \forall \beta \in \mathbf{R}_{+}^{J} .
$$

We multiply (27) by the denominator $1+\pi^{*}(\bar{\alpha}, y)$ and we add to (29) to obtain the property: $\forall \varepsilon>0, \exists \bar{x} \in \Delta(I)$ and $\bar{\alpha} \in \mathbf{R}_{+}^{I}$ such that

$$
v_{1} \leq \frac{f(\bar{x}, y)+f^{*}(\bar{\alpha}, y)+f^{*}(\bar{x}, \beta)}{1+\pi^{*}(\bar{\alpha}, y)+\pi^{*}(\bar{x}, \beta)}+\varepsilon, \quad \forall y \in \Delta(J), \beta \in \mathbf{R}_{+}^{J}
$$

which implies (24), hence the result.

\subsection{General evaluation}

In this section we consider general evaluation probabilities $\mu=\left(\mu_{m}\right)$ on $\mathbb{I}^{\star}$ such that $\left(\mu_{m}\right)$ is non increasing: this later assumption is implicit in all the result below. The payoff corresponding to an evaluation $\mu$ is $\sum_{m} \mu_{m} h_{m}$, where $h_{m}$ is the payoff at stage $m$ described above. We denote by $v_{\mu}$ the value of this game. Our aim is to show that the $v_{\mu}$ have a limit as the "size" of the evaluation probability, i.e., $\pi(\mu):=\mu_{1}=\sup _{m} \mu_{m}$, tends to 0 .

Theorem 23 As $\pi(\mu) \rightarrow 0$, $v_{\mu}$ converges to $v$ given by

$$
v=\operatorname{val}_{((x, \alpha),(y, \beta)) \in\left(\Delta(I) \times \mathbf{R}_{+}^{I}\right) \times\left(\Delta(J) \times \mathbf{R}_{+}^{J}\right)} \frac{f(x, y)+f^{*}(\alpha, y)+f^{*}(x, \beta)}{1+\pi^{*}(\alpha, y)+\pi^{*}(x, \beta)}
$$

The proof requires several steps. The main idea is, as before, to embed the original problem into a game on $[0,1]$. Recall that $\mu$ induces a partition $\Pi=\left\{t_{m}\right\}$ of $[0,1]$ with $t_{0}=0$ and $t_{m}=\sum_{k=1}^{m} \mu_{k}$ for $m \geq 1$. Let us denote by $W_{\mu}\left(t_{m}\right)$ the value of the game starting at time $t_{m}$, i.e. with evaluation $\mu_{m+k}$ for the payoff $h_{k}$ at stage $k$. Note that $W_{\mu}$ is actually given by $W_{\mu}(1)=0$ and the recursive formula:

$$
W_{\mu}\left(t_{m}\right)=\operatorname{val}_{(x, y) \in \Delta(I) \times \Delta(J)}\left[\mu_{m+1} f(x, y)+\pi(x, y) W_{\mu}\left(t_{m+1}\right)+\left(1-t_{m+1}\right) f^{*}(x, y)\right] .
$$

Recall that, under our assumption on the monotonicity of the $\left(\mu_{m}\right)$, the (linear interpolation of) $W_{\mu}$ is $C$-Lipschitz continuous in $[0,1]$, where $C$ only depends on the bounds on the payoff (see Lemma 1). Let us set, for any $(t, a, b, x, \alpha, y, \beta) \in[0,1] \times \mathbf{R} \times \mathbf{R} \times \Delta(I) \times \mathbf{R}_{+}^{I} \times \Delta(J) \times \mathbf{R}_{+}^{J}$,

$$
h(t, a, b, x, \alpha, y, \beta)=\frac{f(x, y)+(1-t)\left[f^{*}(\alpha, y)+f^{*}(x, \beta)\right]-\left[\pi^{*}(\alpha, y)+\pi^{*}(x, \beta)\right] a+b}{1+\pi^{*}(\alpha, y)+\pi^{*}(x, \beta)}
$$

We define the lower and upper Hamiltonian of the game as

$$
H^{-}(t, a, b)=\sup _{(x, \alpha) \in \Delta(I) \times \mathbf{R}_{+}^{I}} \inf _{(y, \beta) \in \Delta(J) \times \mathbf{R}_{+}^{J}} h(t, a, b, x, \alpha, y, \beta)
$$


and

$$
H^{+}(t, a, b)=\inf _{(y, \beta) \in \Delta(J) \times \mathbf{R}_{+}^{J}} \sup _{(x, \alpha) \in \Delta(I) \times \mathbf{R}_{+}^{I}} h(t, a, b, x, \alpha, y, \beta)
$$

The variational characterization of any cluster point $U$ of the family $W_{\mu}$ as $\pi(\mu) \rightarrow 0$ uses the following properties: for all $t \in[0,1)$ and any $C^{1}$ function $\phi:[0,1] \rightarrow \mathbf{R}$ :

- R1: If $U(\cdot)-\phi(\cdot)$ admits a global maximum at $t \in[0,1)$ then $H^{-}\left(t, U(t), \phi^{\prime}(t)\right) \geq 0$.

- R2: If $U(\cdot)-\phi(\cdot)$ admits a global minimum at $t \in[0,1)$ then $H^{+}\left(t, U(t), \phi^{\prime}(t)\right) \leq 0$.

Lemma 24 Any accumulation point $U(\cdot)$ of $W_{\mu}(\cdot)$ satisfies $\boldsymbol{R} \mathbf{1}$ and $\boldsymbol{R} 2$.

Proof. Let us prove the first variational inequality, the second being obtained by symmetry.

Let $t$ be such that $U(\cdot)-\phi(\cdot)$ admits a global maximum at $t \in[0,1)$. Adding $(\cdot-t)^{2}$ to $\phi$ if necessary, we can assume that this global maximum is strict.

Let $\mu^{n}=\left\{\mu_{m}^{n}\right\}$ be a sequence of evaluation probabilities on $\mathbb{I N}^{\star}$ such that $\pi\left(\mu^{n}\right) \rightarrow 0$ and $W_{n}:=W_{\mu^{n}}$ converges to $U$. Let $t_{\theta(n)}^{n}$ be a global maximum of $W_{n}(\cdot)-\phi(\cdot)$ over the set $\left\{t_{m}^{n}\right\}$. Then, $t_{\theta(n)}^{n} \rightarrow t$. Since $t<1$, for $n$ large enough $\theta(n)+1$ is well defined and from (32) we have

$$
W_{n}\left(t_{\theta(n)}^{n}\right)=\max _{x \in \Delta(I)} \min _{y \in \Delta J)}\left[\mu_{\theta(n)+1}^{n} f(x, y)+\pi(x, y) W_{n}\left(t_{\theta(n)+1}^{n}\right)+\left(1-t_{\theta(n)+1}^{n}\right) f^{*}(x, y)\right] .
$$

Let $x_{n}$ be optimal for player 1 in the above formula. By compactness one can assume that $x_{n}$ converges to some $\bar{x}$ (up to a subsequence).

To simplify the notations, we set:

$$
\nu_{n}=\mu_{\theta(n)+1}^{n}, s_{n}=t_{\theta(n)}^{n}, s_{n}^{\prime}=t_{\theta(n)+1}^{n}=s_{n}+\nu_{n}, \alpha_{n}=\frac{x_{n}}{\nu_{n}}
$$

Given $j \in J$ we have:

$$
W_{n}\left(s_{n}\right) \leq \nu_{n} f\left(x_{n}, j\right)+\pi\left(x_{n}, j\right) W_{n}\left(s_{n}^{\prime}\right)+\left(1-s_{n}^{\prime}\right) f^{*}\left(x_{n}, j\right)
$$

Using the fact that $W_{n}(\cdot)-\phi(\cdot)$ has a global maximum at $s_{n}$ the above inequality can be rephrased as

$$
0 \leq f\left(x_{n}, j\right)+\frac{\phi\left(s_{n}^{\prime}\right)-\phi\left(s_{n}\right)}{\nu_{n}}-\pi^{*}\left(\alpha_{n}, j\right) W_{n}\left(s_{n}^{\prime}\right)+\left(1-s_{n}^{\prime}\right) f^{*}\left(\alpha_{n}, j\right) .
$$

We divide this inequality by $1+\pi^{*}\left(\alpha_{n}, j\right)$ so that the quotient is uniformly bounded. Hence, going to the limit and taking subsequences for each $j$ one after the other, we obtain that: for any $\varepsilon>0$ there exists $\bar{\alpha}$ such that:

$$
0 \leq \frac{f(\bar{x}, j)+\phi^{\prime}(t)-\pi^{*}(\bar{\alpha}, j) U(t)+(1-t) f^{*}(\bar{\alpha}, j)}{1+\pi^{*}(\bar{\alpha}, j)}+\varepsilon, \forall j \in J
$$

The same inequality holds for any $y \in \Delta(J)$ instead of $j$ by linearity.

Now $\bar{x}$ is optimal for $U(t)$ leading to

$$
0 \leq(1-t) f^{*}(\bar{x}, y)-\pi^{*}(\bar{x}, y) U(t), \quad \forall y \in \Delta(J)
$$

and by linearity the same inequality holds for any $\beta \in \mathbf{R}_{+}^{J}$.

We multiply (34) by $\left(1+\pi^{*}(\bar{\alpha}, y)\right)$ and we add (35) to obtain, $\forall y \in \Delta(J), \forall \beta \in \mathbf{R}_{+}^{J}$

$$
0 \leq \frac{f(\bar{x}, y)+\phi^{\prime}(t)-\left(\pi^{*}(\bar{\alpha}, y)+\pi^{*}(\bar{x}, \beta)\right) U(t)+(1-t)\left(f^{*}(\bar{\alpha}, y)+f^{*}(\bar{x}, \beta)\right)}{1+\pi^{*}(\bar{\alpha}, y)+\pi^{*}(\bar{x}, \beta)}+\varepsilon .
$$


Hence for any $\varepsilon>0$, there exists $\bar{x} \in \Delta(I), \bar{\alpha} \in \mathbf{R}_{+}^{I}$ such that $\forall y \in \Delta(J), \forall \beta \in \mathbf{R}_{+}^{J}$

$$
h\left(t, U(t), \phi^{\prime}(t), \bar{x}, \bar{\alpha}, y, \beta\right)+\varepsilon \geq 0
$$

which implies $H^{-}\left(t, U(t), \phi^{\prime}(t)\right) \geq 0$.

Next we show a comparison principle:

Lemma 25 Let $U_{1}$ and $U_{2}$ be two continuous functions satisfying $\boldsymbol{R} 1-\boldsymbol{R} 2$ and $U_{1}(1) \leq U_{2}(1)$. Then $U_{1} \leq U_{2}$ on $[0,1]$.

Proof. By contradiction, suppose that there is some $t \in[0,1]$ such that $U_{1}(t)>U_{2}(t)$. Then, for $\gamma>0$ sufficiently small,

$$
\max _{t \in[0,1]}\left[U_{1}(t)-U_{2}(t)+\gamma(t-1)\right]=\delta>0
$$

Let $\varepsilon>0$ and set

$$
\delta(\varepsilon)=\max _{(t, s) \in[0,1] \times[0,1]}\left[U_{1}(t)-U_{2}(s)-\frac{(t-s)^{2}}{2 \varepsilon}+\gamma(s-1)\right] .
$$

Let $\left(t_{\varepsilon}, s_{\varepsilon}\right)$ be a maximum point in the above expression. Then, $\delta(\varepsilon) \rightarrow \delta$ as $\varepsilon \rightarrow 0$ and, for $\varepsilon$ sufficiently small, $t_{\varepsilon}<1$ and $s_{\varepsilon}<1$ because $U_{1}(1) \leq U_{2}(1)$. From standard arguments, $t_{\varepsilon}-s_{\varepsilon} \rightarrow 0$ as $\varepsilon \rightarrow 0$.

Since the map $U_{1}(t)-\frac{\left(t-s_{\varepsilon}\right)^{2}}{2 \varepsilon}$ has a global maximum at $t_{\varepsilon} \in[0,1)$, we have by condition $\mathbf{R} 1$ that

$$
H^{-}\left(t_{\varepsilon}, U_{1}\left(t_{\varepsilon}\right), \frac{t_{\varepsilon}-s_{\varepsilon}}{\varepsilon}\right) \geq 0
$$

In the same way, since the map $s \rightarrow U_{2}(s)+\frac{\left(t_{\varepsilon}-s\right)^{2}}{2 \varepsilon}-\gamma(s-1)$ has a global minimum at $s_{\varepsilon}$, we have by condition $\mathbf{R} 2$ that

$$
H^{+}\left(s_{\varepsilon}, U_{2}\left(s_{\varepsilon}\right), \frac{t_{\varepsilon}-s_{\varepsilon}}{\varepsilon}+\gamma\right) \leq 0
$$

To simplify the expressions, let us set $U_{1}^{\varepsilon}=U_{1}\left(t_{\varepsilon}\right), U_{2}^{\varepsilon}=U_{2}\left(s_{\varepsilon}\right)$ and $b_{\varepsilon}=\frac{t_{\varepsilon}-s_{\varepsilon}}{\varepsilon}$. From (37) and (38) there exists $\left(x_{\varepsilon}, \alpha_{\varepsilon}\right) \in \Delta(I) \times \mathbf{R}_{+}^{I}$ such that:

$$
0 \leq \varepsilon^{2}+\inf _{(y, \beta)} h\left(t_{\varepsilon}, U_{1}^{\varepsilon}, b_{\varepsilon}, x_{\varepsilon}, \alpha_{\varepsilon}, y, \beta\right)
$$

and $\left(y_{\varepsilon}, \beta_{\varepsilon}\right) \in \Delta(J) \times \mathbf{R}_{+}^{J}$ such that

$$
0 \geq-\varepsilon^{2}+\sup _{(x, \alpha)} h\left(s_{\varepsilon}, U_{2}^{\varepsilon}, b_{\varepsilon}+\gamma, x, \alpha, y_{\varepsilon}, \beta_{\varepsilon}\right) .
$$

Then, in view of the definition of $h$, we have

$$
\begin{aligned}
2 \varepsilon^{2} & \geq h\left(s_{\varepsilon}, U_{2}^{\varepsilon}, b_{\varepsilon}+\gamma, x_{\varepsilon}, \alpha_{\varepsilon}, y_{\varepsilon}, \beta_{\varepsilon}\right)-h\left(t_{\varepsilon}, U_{1}^{\varepsilon}, b_{\varepsilon}, x_{\varepsilon}, \alpha_{\varepsilon}, y_{\varepsilon}, \beta_{\varepsilon}\right) \\
& \geq \frac{\left(t_{\varepsilon}-s_{\varepsilon}\right)\left[f^{*}\left(\alpha_{\varepsilon}, y_{\varepsilon}\right)+f^{*}\left(x_{\varepsilon}, \beta_{\varepsilon}\right)\right]-\left[\pi^{*}\left(\alpha_{\varepsilon}, y_{\varepsilon}\right)+\pi^{*}\left(x_{\varepsilon}, \beta_{\varepsilon}\right)\right]\left(U_{\varepsilon}^{2}-U_{\varepsilon}^{1}\right)+\gamma}{1+\pi^{*}\left(\alpha_{\varepsilon}, y_{\varepsilon}\right)+\pi^{*}\left(x_{\varepsilon}, \beta_{\varepsilon}\right)}
\end{aligned}
$$


Now we use $U_{\varepsilon}^{1}-U_{\varepsilon}^{2} \geq \delta(\varepsilon)$ to obtain

$$
\begin{aligned}
2 \varepsilon^{2} & \geq \frac{\left(t_{\varepsilon}-s_{\varepsilon}\right)\left[f^{*}\left(\alpha_{\varepsilon}, y_{\varepsilon}\right)+f^{*}\left(x_{\varepsilon}, \beta_{\varepsilon}\right)\right]+\left[\pi^{*}\left(\alpha_{\varepsilon}, y_{\varepsilon}\right)+\pi^{*}\left(x_{\varepsilon}, \beta_{\varepsilon}\right)\right] \delta(\varepsilon)+\gamma}{1+\pi^{*}\left(\alpha_{\varepsilon}, y_{\varepsilon}\right)+\pi^{*}\left(x_{\varepsilon}, \beta_{\varepsilon}\right)} \\
& \geq \frac{\left(t_{\varepsilon}-s_{\varepsilon}\right)\left[f^{*}\left(\alpha_{\varepsilon}, y_{\varepsilon}\right)+f^{*}\left(x_{\varepsilon}, \beta_{\varepsilon}\right)\right]}{1+\pi^{*}\left(\alpha_{\varepsilon}, y_{\varepsilon}\right)+\pi^{*}\left(x_{\varepsilon}, \beta_{\varepsilon}\right)}+\min \{\delta(\varepsilon), \gamma\}
\end{aligned}
$$

Since $t_{\varepsilon}-s_{\varepsilon} \rightarrow 0$ and the quotient $\frac{f^{*}\left(\alpha_{\varepsilon}, y_{\varepsilon}\right)+f^{*}\left(x_{\varepsilon}, \beta_{\varepsilon}\right)}{1+\pi^{*}\left(\alpha_{\varepsilon}, y_{\varepsilon}\right)+\pi^{*}\left(x_{\varepsilon}, \beta_{\varepsilon}\right)}$ remains bounded as $\varepsilon \rightarrow 0$, we get $0 \geq \min \{\delta, \gamma\}$, which is impossible.

To summarize, we now know that the family $\left(W_{\mu}\right)$ has a unique accumulation point $U$ and that this accumulation point is the unique continuous map satisfying R1-R2 and $U(1)=0$. The next Lemma, which characterizes the limit function $U$, completes the proof of Theorem 23:

Lemma 26 Let $U(\cdot)$ be the unique continuous solution to $\boldsymbol{R} 1-\boldsymbol{R} 2$ with $U(1)=0$. Then $U(t)=$ $(1-t) v$ where $v$ is given by (31).

Proof. Let us first show that $U$ is homogeneous in time. This could be obtained by the fact that $U$ is the limit of the $W_{\pi}$, but we give here a direct argument. For this we prove that $U_{\lambda}(t):=\frac{1}{\lambda} U(\lambda t+(1-\lambda))$ equals $U(t)$ for any $t \in[0,1]$ and any $\lambda \in(0,1)$ by showing that $U_{\lambda}$ satisfies R1-R2 and $U_{\lambda}(1)=0$. The last point being obvious, let us check for instance that R1 holds for $U_{\lambda}$. Since $U$ satisfies $\mathbf{R} 1$ for $H^{-}, U_{\lambda}$ satisfies $\mathbf{R} 1$ for $H_{\lambda}^{-}$given by

$$
H_{\lambda}^{-}(t, a, b)=H^{-}(\lambda t+(1-\lambda), \lambda a, b)
$$

So we just have to show that $H_{\lambda}^{-}(t, a, b) \geq 0$ implies $H^{-}(t, a, b) \geq 0$. Assume that $H_{\lambda}^{-}(t, a, b) \geq 0$. Then, for any $\varepsilon>0$, there exists $(x, \alpha) \in \Delta(I) \times \mathbf{R}_{+}^{I}$ such that, for all $(y, \beta) \in \Delta(J) \times \mathbf{R}_{+}^{J}$,

$$
-\varepsilon \leq \frac{f(x, y)+(1-(\lambda t+(1-\lambda)))\left[f^{*}(\alpha, y)+f^{*}(x, \beta)\right]-\left[\pi^{*}(\alpha, y)+\pi^{*}(x, \beta)\right] \lambda a+b}{1+\pi^{*}(\alpha, y)+\pi^{*}(x, \beta)}
$$

Setting $\alpha^{\prime}=\lambda \alpha$ and $\beta^{\prime}=\lambda \beta$ we get

$$
-\frac{\varepsilon}{\lambda} \leq \frac{f(x, y)+(1-t)\left[f^{*}\left(\alpha^{\prime}, y\right)+f^{*}\left(x, \beta^{\prime}\right)\right]-\left[\pi^{*}\left(\alpha^{\prime}, y\right)+\pi^{*}\left(x, \beta^{\prime}\right)\right] \lambda a+b}{1+\pi^{*}\left(\alpha^{\prime}, y\right)+\pi^{*}\left(x, \beta^{\prime}\right)}
$$

because

$$
-\frac{\varepsilon\left(1+\pi^{*}(\alpha, y)+\pi^{*}(x, \beta)\right.}{1+\pi^{*}\left(\alpha^{\prime}, y\right)+\pi^{*}\left(x, \beta^{\prime}\right)} \geq-\frac{\varepsilon}{\lambda}
$$

Therefore there exists $\left(x, \alpha^{\prime}\right) \in \Delta(I) \times \mathbf{R}_{+}^{I}$ such that, for all $\left(y, \beta^{\prime}\right) \in \Delta(J) \times \mathbf{R}_{+}^{J}$, one has $h(t, a, b, x, \alpha, y, \beta) \geq-\varepsilon / \lambda$, i.e., $H^{-}(t, a, b) \geq 0$.

Next we identify $v:=U(0)$. From the equation satisfied by $U(t)=(1-t) v$ we have, using $\phi(t)=U(t)$ :

$$
H^{-}(t,(1-t) v,-v) \geq 0 \quad \text { and } \quad H^{+}(t,(1-t) v,-v) \leq 0 \quad \forall t \in[0,1] .
$$

Let us choose $t=0$. Let $\varepsilon>0$ and $(x, \alpha)$ be such that for any $(y, \beta)$

$$
-\varepsilon \leq \frac{f(x, y)+\left[f^{*}(\alpha, y)+f^{*}(x, \beta)\right]-\left[\pi^{*}(\alpha, y)+\pi^{*}(x, \beta)\right] v-v}{1+\pi^{*}(\alpha, y)+\pi^{*}(x, \beta)}
$$

Then

$$
v-\varepsilon \leq \frac{f(x, y)+f^{*}(\alpha, y)+f^{*}(x, \beta)}{1+\pi^{*}(\alpha, y)+\pi^{*}(x, \beta)}
$$


so that

$$
v-\varepsilon \leq \sup _{(x, \alpha)} \inf _{(y, \beta)} \frac{f(x, y)+f^{*}(\alpha, y)+f^{*}(x, \beta)}{1+\pi^{*}(\alpha, y)+\pi^{*}(x, \beta)}
$$

The opposite inequality

$$
v+\varepsilon \geq \inf _{(y, \beta)} \sup _{(x, \alpha)} \frac{f(x, y)+f^{*}(\alpha, y)+f^{*}(x, \beta)}{1+\pi^{*}(\alpha, y)+\pi^{*}(x, \beta)}
$$

can be established in a symmetric way, which completes the proof of the Lemma.

\section{$5 \quad$ Extensions and comments}

\subsection{Non decreasing evaluations}

In stochastic games whith general evaluation, to obtain the same asymptotic limit as the mesh of the partition tends to zero, it is necessary to assume the sequence of evaluation probabilities $\mu^{n}$ on $I^{*}$ to be decreasing: $\mu_{m}^{n} \geq \mu_{m+1}^{n}$. For example, if the stochastic game oscillates deterministically between state 1 and state 2 , the asymptotic occupation measure depends strongly on $\mu^{n}$. For example, if $\mu^{n}$ is decreasing, then asymptotically, both states have a total weight of $1 / 2$. However, if $\left\{\mu_{2 m+1}^{n}\right\}$ is decreasing in $m$ and if $\mu_{2 m}^{n}=\left(\mu_{2 m+1}^{n}\right)^{2}$, then the asymptotic occupation measure puts a total weight of 1 on the state at stage 1 .

However, in all games analyzed in this paper, the monotonicity assumption on $\mu_{m}$ is not necessary: the asymptotic value exists and is the same for all evaluation measures. This is due to the irreversibility of these games. In incomplete information repeated games, the results hold because of two reasons: (1) a player is always better off having a private information (which implies concavity of the value function in $p$ and convexity in $q$ ), and (2) a player has always the possibility to play a non-revealing strategy. Then $V_{\Pi}$ is $C$-Lipschitz continuous: this is the content of Lemma 15.

Consequently, the same proof as for decreasing evaluations applies and so the asymptotic value exists in a strong sense and is characterized as the unique solution of the variational inequalities $P 1$ and $P 2$. A similar argument shows that the same conclusion holds for splitting games.

In absorbing games, this conclusion holds because once the state changes, it is absorbing. The proof is however more tricky. Let $W_{\mu^{n}}\left(t_{k}\right)$ be the value of the game starting at time $t_{k}$. Then:

$$
W_{\mu^{n}}\left(t_{k}\right)=\operatorname{val}_{(x, y) \in \Delta(I) \times \Delta(J)}\left[\mu_{k+1}^{n} f(x, y)+\pi(x, y) W_{\mu^{n}}\left(t_{k+1}\right)+\left(1-t_{k+1}\right) f^{*}(x, y)\right] .
$$

As shown in Lemma 1, monotonicity of $\left(\mu_{m}^{n}\right)$ in $m$ guarantees that $W_{\mu^{n}}$ is $C$-Lipschitz continuous. Without this assumption, it is not clear how to show uniform Lipschitz continuity.

We prove uniform convergence but using different techniques, standard in differential game theory. Namely, consider the Barles-Perthame lower and upper half-relaxed limits. Explicitly, for every $t$, define $W^{+}(t)=\lim \sup _{t_{n} \rightarrow t} W_{\mu^{n}}\left(t^{n}\right)$, and similarly $W^{-}(t)=\liminf t_{t_{n} \rightarrow t} W_{\mu^{n}}\left(t^{n}\right)$. Then, $W^{+}(t)$ is upper-semi-continuous and $W^{-}(t)$ is lower-semi-continuous. A proof similar to the one given for the decreasing case (with only small modifications) shows that: (1) $W^{+}$satisfies R1, (2) $W^{-}$satisfies $\mathbf{R 2}$, and (3) any upper-semi-continuous function satisfying $\mathbf{R} \mathbf{1}$ is smaller than any lower-semi-continuous function satisfying R2 (whenever they agree on the terminal condition). This implies uniform convergence and uniqueness of the limit.

Observe also that in the three classes of games analyzed in this paper, the existence of the asymptotic value in a strong sense (for all evaluations not necessarily decreasing) is new. Actually, the existence of the uniform value (as in absorbing games, Kohlberg (1974) [5] ) only implies the same asymptotic value for all decreasing evaluations. 
A natural question arises: what are the other classes of repeated games for which the asymptotic value is the same for all evaluations? Clearly, this is quite different from the existence of a uniform value. In the example above (stochastic game alternating between states 1 and 2), a uniform value exists but the asymptotic value depends on the sequence of evaluations. In incomplete information repeated games and in splitting games, the uniform value does not exist while there is a "strong" asymptotic value.

\subsection{Other extensions}

More general splitting games. Upper- and lower half-relaxed limits have been used in Laraki [6] to show the existence of the asymptotic value in discounted splitting games when $P$ and $Q$ are not product of simplexes. Without this assumption, the equi-continuity of the family of discounted values with respect to $p$ and $q$ is not guaranteed. Combining the technique in Laraki [6] and the continuous time approach allow to show the existence of the asymptotic value for all evaluations under the same general assumptions as the one in Laraki [6].

Repeated games with public random duration. Neyman and Sorin [13] studied repeated games with random duration. Those are games in which the weight $\mu_{m}$ of period $m$ follows a stochastic process. In our model, this weight is deterministic. Neyman and Sorin [13] show that when the uniform value exists, then the asymptotic value exists for all random duration. It is plausible to prove existence of an asymptotic value in repeated games with random duration using similar tools. The difference would be in the recursive equation: an additional expectation should be added since the time $t_{k+1}$ at which the continuation game will start is random and not deterministic.

Repeated games with incomplete information: the dependent case. The result of Mertens and Zamir [11] holds in a more general framework in which the private information of the players on $k \in K$ may be correlated. However one can write a recursive equation on the state space $\Delta(K)$. Consequently, the same proof as in the independent case allows to prove existence, uniqueness and characterization of the asymptotic value, for all evaluation coefficients $\mu$.

\subsection{Conclusion}

The main contribution of this approach is to provide a unified treatment of the asymptotic analysis of the value of repeated games:

- it applies to all evaluations and shows the interest of the limiting game played on $[0,1]$. Further research will be devoted to a formal construction and to the analysis of optimal strategies,

- it allows to treat incomplete information games as well as absorbing games. We strongly believe that similar tools will allow to analyze more general classes,

- it shows that technics introduced in differential games where the dynamics on the state are smooth can be used in a repeated game framework. On the other hand, the stationary aspect of the payoff functions in repeated games is no longer necessary to obtain asymptotic properties.

\section{References}

[1] Aumann R.J. and M. Maschler (1995). Repeated Games with Incomplete Information, M.I.T. Press.

[2] Barles, G. and Souganidis, P. E. (1991) Convergence of approximation schemes for fully nonlinear second order equations. Asymptotic Anal. 4, 3, 271-283. 
[3] Bewley T. and Kohlberg E. (1976a). The Asymptotic Theory of Stochastic Games. Mathematics of Operations Research, 1, 197-208.

[4] Bewley T. and Kohlberg E. (1976b). The Asymptotic Solution of a Recursion Equation Occurring in Stochastic Games. Mathematics of Operations Research, 1, 321-336.

[5] Kohlberg E. (1974). Repeated Games with Absorbing States. Annals of Statistics, 2, 724-738.

[6] Laraki R. (2001a). The Splitting Game and Applications. International Journal of Game Theory, 30, 359-376.

[7] Laraki R. (2001b). Variational Inequalities, System of Functional Equations, and Incomplete Information Repeated Games. SIAM J. Control and Optimization, 40, 516-524.

[8] Laraki R. (2002). Repeated Games with Lack of Information on One Side : the Dual Differential Approach. Mathematics of Operations Research, 27, 419-440.

[9] Laraki R. (2004). On the Regularity of the Convexification Operator on a Compact Set. Journal of Convex Analysis, 11, 209-234.

[10] Laraki R. (2010). Explicit Formulas for Repeated Games with Absorbing States. International Journal of Game Theory, 39, 53-69.

[11] Mertens J.-F. and S. Zamir (1971). The Value of Two-Person Zero-Sum Repeated Games with Lack of Information on Both Sides. International Journal of Game Theory, 1, 39-64.

[12] Mertens J.-F., S. Sorin and S. Zamir (1994). Repeated Games. CORE DP 9420-22.

[13] Neyman A. and S. Sorin (2010). Repeated games with public uncertain duration process, International Journal of Game Theory, 39, 29-52.

[14] Rosenberg D. (2000). Zero-Sum Absorbing Games with Incomplete Information on One Side: Asymptotic Analysis. SIAM J. Control and Optimization, 39, 557-597.

[15] Rosenberg D. and S. Sorin (2001). An Operator Approach to Zero-Sum Repeated Games. Israel Journal of Mathematics, 121, 221-246.

[16] Rosenberg D. and N. Vieille (2001). The MaxMin of Recursive Games with Incomplete Information on One Side. Mathematics of Operations Research, 25, 23-35.

[17] Shapley L. S. (1953). Stochastic Games. Proceedings of the National Academy of Sciences of the U.S.A., 39, 1095-1100.

[18] Sorin S. (1984). "Big Match" with Lack of Information on One Side, Part I. International Journal of Game Theory, 13, 201-255.

[19] Sorin S. (1985). "Big Match" with Lack of Information on One Side, Part II. International Journal of Game Theory, 14, 173-204.

[20] Sorin S. (2002). A First Course on Zero-Sum Repeated Games. Springer.

[21] Sorin S. (2005). New Approaches and Recent Advances in Two-Person Zero-Sum Repeated Games. Advances in Dynamic Games, A. Nowak and K. Szajowski (eds.), Annals of the ISDG, 7, Birkhauser, 67-93.

[22] Vieille N. (1992). Weak Approachability. Mathematics of Operations Research, 17, 781-791. 